\title{
Exponential decay rates for a full von Karman thermoelastic system with nonlinear thermal coupling.
}

\author{
Assia Benabdallah* Irena Lasiecka ${ }^{\dagger}$
}

\begin{abstract}
The full von Karman system accounting for in plane acceleration and nonlinear thermal effects is considered. The results obtained in this paper: (i) wellposedness of regular and weak (finite energy) solutions, (ii) uniform decay rates of energy function, extend those obtained earlier in [7] for a more restrictive model which does not account for the nonlinear thermal coupling.
\end{abstract}

\section{Introduction}

We consider a model describing nonlinear oscillations of a plate subjected to thermal effects and accounting for in plane accelerations. The resulting model consists of an elastodynamic system coupled nonlinearly with the Kirchoff plate equation and two heat equations. As such, it is referred to as full von Karman thermoelastic system.

The linear models of thermoelasticity were considered in $[31,32,33,30]$. The nonlinear full von Karman system of thermoelasticity has been considered in [6] and [7]. However, these models accounted for linear thermal coupling. A more accuarate model involves a nonlinear thermal coupling, in addition to the full nonlinear mechanical coupling. The main aim of this paper is to provide stability analysis for this more general model with nonlinear thermal coupling. Precise derivation and physical justification of the model is given in [6]. We begin with the PDE equations describing the model.

The variables $w$ and $u=\left(u_{1}, u_{2}\right)$ represent, respectively, the vertical and in plane displacement of a thin plate occupying a two dimensional domain $\Omega$ with sufficiently smooth boundary $\Gamma$. The variables $\theta$ and $\phi$ describe the temperature affecting vertical displacement and horizontal displacements respectively. We shall decompose the vector $u \in\left[H^{1}(\Omega)\right]^{2}$ into rotational and solenoidal part $u=\nabla p+$ curlq, where $p \equiv-A_{D}^{-1}($ divu $)$ with $A_{D}$ denoting the negative Laplace'a operator subject to zero

\footnotetext{
* Laboratoire de Calcul Scientifique et UMR 6623, Université de Franche Comté, 25030 Besançon, France. e-mail: assiamath.univ-fcomte.fr

${ }^{\dagger}$ Department of Mathematics, University of Virginia, Charlottesville, Va 22903. e-mail: il2vvirginia.edu
} 
Dirichlet boundary conditions. With the above notation, the governing equations to be considered are given by:

$$
\begin{gathered}
u_{t t}+b \operatorname{curlq}_{t}-\operatorname{div}[\mathcal{C}[\boldsymbol{\epsilon}(u)+f(\nabla w)]]+\nabla \phi=0 \text { in } \Omega \times(0, \infty) \\
{[I-\gamma \Delta] w_{t t}+D \Delta^{2} w} \\
-\operatorname{div}[\mathcal{C}[\boldsymbol{\epsilon}(u)+f(\nabla w)] \nabla w+\phi \nabla w]+\Delta \theta=0 \text { in } \Omega \times(0, \infty)
\end{gathered}
$$

with the Dirichlet boundary conditions $u=w=\nabla w=0$ on $\Gamma \times(0, \infty)$. The temperature is governed by the following system of equations

$$
\begin{aligned}
\phi_{t}=\Delta \phi-\text { divu }_{t}+\nabla w \nabla w_{t} ; & \text { in } \Omega \times(0, \infty) \\
\theta_{t}=\Delta \theta+\Delta w_{t} & \text { in } \Omega \times(0, \infty)
\end{aligned}
$$

with the Dirichlet zero boundary conditions $\theta=\phi=0$ on $\Gamma \times(0, \infty)$.

With ( 1) and ( 2) we associate the initial conditions

$$
\begin{gathered}
u(0)=u_{0}, u_{t}(0)=u_{1}, w(0)=w_{0}, w_{t}(0)=w_{1} ; \\
\theta(0)=\theta_{0}, \phi(0)=\phi_{0} \text { in } \Omega
\end{gathered}
$$

$D$ represents the flexural rigidity, the constant $0<\mu<1 / 2$ is Poisson's modulus and the constant $\gamma \geq 0$ is proportional to the thickness of the plate.

The fourth order tensor $\mathcal{C}$ is defined by

$$
\mathcal{C}(\boldsymbol{\epsilon}) \equiv \frac{E}{(1-2 \mu)(1+\mu)}[\mu \text { trace } \boldsymbol{\epsilon} I+(1-2 \mu) \boldsymbol{\epsilon}]
$$

with the strain tensor given by $\epsilon(u) \equiv 1 / 2\left(\nabla u+\nabla^{T} u\right)$. It can be easily verified that the tensor $\mathcal{C}$ is symmetric and strictly positive. The function $f$ is given by

$$
f(s) \equiv(1 / 2) s \times s ; s \in R^{2}
$$

The main results related to the wellposedness/regularity properties of the solutions are formulated below.

\section{Theorem 1.1 Assume that $\gamma>0$.}

- (1) Weak solutions. There exists a unique, global solution of finite energy. This is to say that for any initial data

$$
u_{0}, u_{1}, \theta_{0}, \phi_{0} \in\left[H_{0}^{1}(\Omega)\right]^{2} \times\left[L_{2}(\Omega)\right]^{2} \times\left[L_{2}(\Omega)\right]^{2} ; w_{0}, w_{1} \in H_{0}^{2}(\Omega) \times H_{0}^{1}(\Omega),
$$

there exists a unique solution (of the corresponding variational form (12))

$$
\begin{gathered}
(u, w, \theta, \phi) \in C\left(0, T ;\left[H^{1}(\Omega)\right]^{2} \times H^{2}(\Omega) \times\left[L_{2}(\Omega)\right]^{2}\right) ; \\
\left(u_{t}, w_{t}\right) \in C\left(0, T ;\left[L_{2}(\Omega)\right]^{2} \times H^{1}(\Omega)\right)
\end{gathered}
$$

where $T>0$ is arbitrary. 
- (2) Regular solutions. For any initial data subject to the regularity in part (1) and in addition

$$
\begin{gathered}
u_{0}, u_{1} \in\left[H^{2}(\Omega)\right]^{2} \times\left[H_{0}^{1}(\Omega)\right]^{2} ; w_{0}, w_{1} \in H^{3}(\Omega) \times H_{0}^{2}(\Omega) \\
\theta_{0}, \phi_{0} \in H_{0}^{1}(\Omega) \cap H^{2}(\Omega)
\end{gathered}
$$

there exists a unique, global solution

$$
\begin{aligned}
& (u, w, \theta, \phi) \in C\left(0, T ;\left[H^{2}(\Omega)\right]^{2} \times H^{3}(\Omega) \times\left[H^{2}(\Omega)\right]^{2}\right) \\
& \left(u_{t}, w_{t}, \phi_{t}, \theta_{t}\right) \in C\left(0, T ;\left[H^{1}(\Omega)\right]^{2} \times H^{2}(\Omega) \times\left[L_{2}(\Omega)\right]^{2}\right)
\end{aligned}
$$

where $T>0$ is arbitrary.

Moreover, regular solutions depend continuously on the initial data in the topology of regular solutions (as above).

\section{Assume that $\gamma=0$.}

- (3) For any initial data

$$
u_{0}, u_{1}, \theta_{0}, \phi_{0} \in\left[H_{0}^{1}(\Omega)\right]^{2} \times\left[L_{2}(\Omega)\right]^{2} \times\left[L_{2}(\Omega)\right]^{2} ; w_{0}, w_{1} \in H_{0}^{2}(\Omega) \times L_{2}(\Omega),
$$

there exists a unique finite energy solution

$$
\begin{gathered}
(u, w, \theta, \phi) \in C\left(0, T ;\left[H^{1}(\Omega)\right]^{2} \times H^{2}(\Omega) \times\left[L_{2}(\Omega)\right]^{2}\right) ; \\
\left(u_{t}, w_{t}\right) \in C\left(0, T ;\left[L_{2}(\Omega)\right]^{2} \times L_{2}(\Omega)\right)
\end{gathered}
$$

with the additional regularity

$$
\left(w, w_{t}\right) \in L_{2}\left(0, T ; H^{3}(\Omega) \times H^{1}(\Omega)\right) .
$$

where $T>0$ is arbitrary. The solutions depend continuously on the initial data in the energy norm.

Remark 1.2 The main dificulty in proving the wellposedness (particularly the uniqueness of finite energy solutions, which is critical for our stability results) is related to the fact that the nonlinear terms in the equation are not bounded on the "energy" space (this is due to the lack of appropriate Sobolev's embeddings in the twodimensional case). This precludes applicability of standard methods in nonlinear analysis. 
The main aim of this paper is to establish exponential decay rates for the energy associated with weak solutions.

Uniform decay rates for regular solutions to full von Karman system, without the thermal components, were recently proved for the models with additional mechanical dissipation added on the boundary [31, 32, 33, 40, 26]. On the other hand, it became known recently that the thermal dissipation alone causes the uniform decay of energy in thermoelastic plates regardless the presence of inertial terms (ie $\gamma>0$ or $\gamma=0$ ) $[3,4,5]$. [Exponential decay rates for the energy of linear thermoelastic plates with $\gamma=0$ (analytic case) have been known for some time [see [24, 41, 42, 37, 39]]. Similar results have been extended to several scalar nonlinear plate equations accounting for thermal components $[2,11]$. The one-dimensional full von Karman system accounting for in plane accelerations, with $\gamma>0$, has been treated recently in [9]. Exponential decay rates for the two-dimensional von Karman system with linear thermal coupling were obtained in [7].

The main goal of this paper is to prove the exponential decay rates for all values of the parameter $\gamma \geq 0$ and for all weak solutions to the two-dimensional full von Karman system with nonlinear thermal coupling.

It should be pointed out that the presence of the nonlinear thermal coupling complicates the analysis in a rather substantial manner. In addition to unbounded, with respect to the energy function, nonlinear terms appearing in the mechanical equations we have unbounded nonlinear contribution entering the thermal equation. This causes some major difficulties and the resulting analysis is more subtle.

To state our main result, we define the energy functional associated with the plate model ( 1) and given by

$$
E_{\gamma}(t)=E_{k, \gamma}(t)+E_{p}(t)
$$

with the kinetic energy

$$
E_{k, \gamma}(t)=\int_{\Omega}\left|u_{t}\right|^{2}+w_{t}^{2}+\gamma\left|\nabla w_{t}\right|^{2} d \Omega
$$

and the potential energy:

$$
E_{p}(t)=\int_{\Omega}[\mathcal{C} N(u, w) \cdot N(u, w)]+D|\Delta w|^{2}+|\theta|^{2}+|\phi|^{2} d \Omega
$$

where the stress resultants $N(u, w)$ is given by

$$
N(u, w) \equiv \boldsymbol{\epsilon}(u)+f(\nabla w)
$$

It is well known that $E_{p}(t)$ is topologically equivalent to $H^{2}(\Omega) \times\left[H^{1}(\Omega)\right]^{2} \times$ $\left.L_{2}(\Omega)\right]^{2}$ topology. In particular, the following inequalities resulting from Korn's inequality and Sobolev's imbeddings will be used frequently:

$$
\begin{aligned}
|u|_{H^{1}(\Omega)} & \leq C\left[|N(u, w)|_{L_{2}(\Omega)}+|\nabla w|_{L_{4}(\Omega)}^{2}\right] \\
& \leq C\left[|N(u, w)|_{L_{2}(\Omega)}+|w|_{H^{2}(\Omega)}^{2}\right]
\end{aligned}
$$


Moreover, the following obvious estimates will be used without further mention

$$
\begin{aligned}
|\nabla p|_{H^{1}(\Omega)}+|\operatorname{curl} q|_{H^{1}(\Omega)} & \leq C|u|_{H^{1}(\Omega)} ; \quad\left|\nabla p_{t}\right|_{L_{2}(\Omega)}+\left|\operatorname{curl} q_{t}\right|_{L_{2}(\Omega)} \\
& \leq C\left|u_{t}\right|_{L_{2}(\Omega)}
\end{aligned}
$$

Our main result is:

Theorem 1.3 Let $u, w, \theta, \phi$ be a weak solution to the original system given by (1), (2). Then, the following estimate holds

$$
E_{\gamma}(t) \leq C\left(E_{\gamma}(0)\right) e^{-\omega t} ; \quad t \geq 0
$$

where the constant $\omega>0$ may depend on $E_{\gamma}(0)$, and $\gamma>0$.

When $\gamma=0$ we also obtain:

$$
E_{0}(t) \leq C\left(E_{0}(0)\right) e^{-\omega t} ; \quad t \geq 0
$$

The proof of Theorem 1.3 is based on application of multipliers method introduced in $[3,1]$ (where uniform decay were proved for two-dimensional linear thermoelastic plates) and in [9] (where one-dimensional thermoelastic von Karman model was studied ).

Remark 1.4 1. In the case of linear thermal coupling, an analogous estimate was obtained in [7]. There is, however, one major difference. The estimates given in [7] are independent on the value of the parameter $\gamma>0$. Instead, in our case, the arguments are different for the cases $\gamma>0$ and $\gamma=0$ and it does not seem possible to obtain the uniformity of the decay rates with respect to this parameter. In fact, the analyticity of the model for $\gamma=0$ appears an essential tool in proving the decay rates. This is not the case for the model with the linear thermal coupling.

2. We note that in order to guarantee the decay rates for the energy function, the presence of the damping term curlq $q_{t}$ in the first equation in (1) is necessary. Indeed, this follows from the fact that thermal dissipation in 2-dimensional linear system of elasticity will provide, in general, only strong stability and not the exponential stability- see[35, 22]. For this reason it is necessary to introduce "dissipation" acting at least on the solenoidal part of the horizontal displacement (see [8]). It will be apparent from the proofs carried in the paper, that the results of both Theorem hold without any change if we replace the damping term curl $u_{t}$ by a stronger viscous damping $u_{t}$. In fact, adding viscous damping simplifies some of the arguments considerably.

3. A natural question to ask is whether similar results will hold with the dissipation imposed on $u$ on the boundary rather than in the interior. It is well known that such feedback would provide uniform decay rates for the thermoelastic elastodynamic system- which constitutes one component of the von Karman system. Also, the plate component of von Karman system is exponentially 
stable without any dissipation added [3, 1, 24]. However, the strong nonlinear mechanical coupling between these two components makes the analysis of boundary stabilization very difficult. For the case $\gamma=0$ uniform decay rates with nonlinear boundary feedback were recently established in [27]. However, the case $\gamma>0$ is different and at the present it seems unlikely that such result can be proved for $\gamma>0$ and for the case of boundary dissipation imposed on $u$ only.

The remainder of this paper is devoted to the proofs of Theorems 1.1 and 1.3. Here we note that some of the arguments needed for the proofs of the main results are similar to these presented in [7]. In particular, the proofs of the existence of weak solutions and the regularity of solutions in the case $\gamma>0$ rely on almost identical arguments as these provided in [7]. However, the presence of nonlinear thermal coupling complicates substantially the arguments needed to establish: (i) the uniqueness of weak solutions, (ii) regularity of solutions when $\gamma=0$, and (iii) exponential decay rates. For this reasons, we shall concentrate only on points which are new with respect to [7] and we shall refer to [7] whenever possible.

\section{Proof of Theorem 1}

\section{$2.1 \quad$ Preliminaries}

In what follows we shall use the following notation.

$$
|u|_{\alpha, \Omega} \equiv|u|_{H^{\alpha}(\Omega)},|u|_{\alpha, \Gamma} \equiv|u|_{H^{\alpha}(\Gamma)} ; \quad(u, v)_{\Omega} \equiv(u, v)_{L_{2}(\Omega)} ;\langle u, v\rangle_{\Gamma} \equiv(u, v)_{L_{2}(\Gamma)}
$$

By the same symbol we shall also denote norms/ inner products of two copies of $L_{2}$ or $H^{\alpha}$ spaces. This should not create any confusion, since the meaning will be clear from the context. The constant $C$ is a generic constant, different in various occurences.

We shall begin by writing system $(1)$ in a variational form. To this end let be given two test functions $\xi \in H^{1}(\Omega) \times H^{1}(\Omega)$ and $\psi \in H^{2}(\Omega) \cap H_{0}^{1}(\Omega)$. The von Karman system admits the following variational form:

$$
\begin{gathered}
\left(u_{t t}, \xi\right)_{\Omega}+\left(\text { b curlq }_{t}, \xi\right)_{\Omega}+(\mathcal{C}[\boldsymbol{\epsilon}(u)+f(\nabla w)], \boldsymbol{\epsilon}(\xi))_{\Omega} \\
-\langle\mathcal{C} \boldsymbol{\epsilon}(u) \nu, \xi\rangle_{\Gamma}+(\nabla \phi, \xi)_{\Omega}=0 \\
\left(w_{t t}, \psi\right)_{\Omega}+\gamma\left(\nabla w_{t t}, \nabla \psi\right)_{\Omega}+D(\Delta w, \Delta \psi)_{\Omega} \\
+(\mathcal{C}[\boldsymbol{\epsilon}(u)+f(\nabla w)], \nabla \psi \times \nabla w)_{\Omega} \\
+(\phi \nabla w, \nabla \psi)_{\Omega}-D\left\langle\Delta w, \frac{\partial}{\partial \nu} \psi\right\rangle_{\Gamma}=(\nabla \theta, \nabla \psi)_{\Omega}
\end{gathered}
$$

Note that we have used the boundary conditions satisfied on $\Gamma$.

Later we shall be using variational equality (11) with various choices of test functions $\xi$ and $\psi$. Taking test functions which comply with all boundary conditions imposed by the problem, ie: $\xi \in\left[H_{0}^{1}(\Omega)\right]^{2}$ and $\psi \in H_{0}^{2}(\Omega), \eta, \delta \in H_{0}^{1}(\Omega)$ leads to the following classical variational formulation of the original system (1), (2). 


$$
\begin{array}{r}
\left(u_{t t}, \xi\right)_{\Omega}+\left(b \text { curlq }_{t}, \xi\right)_{\Omega}+(\mathcal{C}[\boldsymbol{\epsilon}(u)+f(\nabla w)], \boldsymbol{\epsilon}(\xi))_{\Omega}+(\nabla \phi, \xi)_{\Omega}=0 \\
\left(w_{t t}, \psi\right)_{\Omega}+\gamma\left(\nabla w_{t t}, \nabla \psi\right)_{\Omega}+D(\Delta w, \Delta \psi)_{\Omega} \\
+(\mathcal{C}[\boldsymbol{\epsilon}(u)+f(\nabla w)], \nabla \psi \times \nabla w)_{\Omega}+(\phi \nabla w, \nabla \psi)_{\Omega}=(\nabla \theta, \nabla \psi)_{\Omega} \\
\left(\phi_{t}, \eta\right)_{\Omega}+(\nabla \phi, \nabla \eta)_{\Omega}-\left(u_{t}, \nabla \eta\right)_{\Omega}-\left(\nabla w \nabla w_{t}, \eta\right)_{\Omega}=0 \\
\left(\theta_{t}, \delta\right)_{\Omega}+(\nabla \theta, \nabla \delta)_{\Omega}+\left(\nabla w_{t}, \nabla \delta\right)_{\Omega}=0
\end{array}
$$

In order to prove the existence of weak solutions we apply a standard nonlinear Galerkin method. By projecting variational form (12) on a family of finite dimensional subspaces we construct semidiscrete approximations of the original system. The appropriate a-priori bounds and passage through the limit with the parameter of the approximation allow to conclude that the semidiscrete approximations converge to a limit which satisfies the variational form given in (12). Since the details of this argument are identical to these provided in [7], they are omitted. Similarily, the analysis of regular solutions (part 2 of Theorem 1.1), based on the analysis of regularity properties of the semidiscrete solutions (uniformly in the parameter of discretization) is the same as that presented in [7], and it will be omitted. The points which definitely require new analysis and where the presence of nonlinear thermal coupling is critical are: uniqueness of weak solutions, the wellposedness for the case $\gamma=0$, and stability analysis. These issues will be elaborated below.

\subsection{Uniqueness of weak solutions - part 1 in Theorem 1}

Here we adapt the method proposed in [43] where Marguerre-Vlasov equations were considered. The same method was used in [26] where wellposedness of full von Karman system without the thermal effects and with nonlinear boundary dissipation is established and in [7] where thermoelastic system with linear thermal coupling was considered. The presence of nonlinear thermal coupling introduces new technical difficulties which require substantail modifications of the arguments. These are provided below.

To begin with, we introduce the operators providing semigroup representation of the thermoelastic system. To this end, let $A_{0}$ be the generator corresponding to the system of elasticity, and $\mathcal{A}$ be the generator corresponding to the biharmonic operator. This is to say:

$$
\begin{gathered}
A_{0} u \equiv-\operatorname{div} \mathcal{C} \boldsymbol{\epsilon}(u) ; \quad D\left(A_{0}\right) \equiv\left[H^{2}(\Omega)\right]^{2} \cap\left[H_{0}^{1}(\Omega)\right]^{2} \\
\mathcal{A} w \equiv \Delta^{2} w ; \quad D(\mathcal{A}) \equiv H_{0}^{2}(\Omega) \cap H^{4}(\Omega)
\end{gathered}
$$

Let $\tilde{u} \equiv u_{1}-u_{2}, \tilde{w} \equiv w_{1}-w_{2}, \tilde{\phi} \equiv \phi_{1}-\phi_{2} ; \tilde{\theta} \equiv \theta_{1}-\theta_{2}$ where $u_{1}, w_{1}, \phi_{1}, \theta_{1}$ and $u_{2}, w_{2}, \phi_{2}, \theta_{2}$ be two potential solutions of finite energy (ie weak solutions) of (1), (2). Using the definitions of operators $A_{0}, \mathcal{A}$ given above and denoting $M \equiv$ $I+\gamma A_{D}$ we rewrite the original PDE as an abstract second order system defined on $\left[D\left(A_{0}\right)\right]^{\prime} \times[D(\mathcal{A})]^{\prime}$ (see [14], [17], [29] ). 


$$
\begin{array}{r}
\tilde{u}_{t t}+b \mathcal{D} \tilde{u}_{t}+A_{0} \tilde{u}+\nabla \tilde{\phi}=f_{1}\left(\tilde{w}, w_{i}\right) \\
M \tilde{w}_{t t}+\mathcal{A} \tilde{w}-A_{D} \tilde{\theta}=f_{2}\left(\tilde{u}, \tilde{w}, u_{i}, w_{i}\right) \\
\tilde{\phi}_{t}+A_{D} \tilde{\phi}+d i v \tilde{u}_{t}=f_{3}\left(\tilde{w}, \tilde{w}_{t}, w_{i}, w_{i t}\right) \\
\tilde{\theta}_{t}+A_{D} \tilde{\theta}+A_{D} \tilde{w}_{t}=0
\end{array}
$$

where, we recall, $\mathcal{D} \in \mathcal{L}\left(\left[L_{2}(\Omega)\right]^{2}\right)$ is given by $\mathcal{D} u \equiv u+\nabla A_{D}^{-1}$ divu, $A_{D}: L_{2}(\Omega) \rightarrow$ $L_{2}(\Omega), A_{D}=-\Delta ; D\left(A_{D}\right)=H^{2}(\Omega) \cap H_{0}^{1}(\Omega)$. The forcing terms $f_{i}$ are defined as follows:

$$
\begin{array}{r}
f_{1}\left(\tilde{w}, w_{i}\right) \equiv \operatorname{div} \mathcal{C}\left[f\left(\nabla w_{1}\right)-f\left(\nabla w_{2}\right)\right] \\
f_{2}\left(\tilde{u}, \tilde{w}, u_{i}, w_{i}\right) \equiv \operatorname{div}\left[\mathcal{C} N\left(u_{1}, w_{1}\right) \nabla w_{1}-\mathcal{C} N\left(u_{2}, w_{2}\right) \nabla w_{2}\right]+\operatorname{div}\left[\tilde{\phi} \nabla w_{1}+\phi_{2} \nabla \tilde{w}\right] \\
f_{3}\left(\tilde{w}, \tilde{w}_{t}, w_{i}, w_{i t}\right) \equiv \nabla \tilde{w} \nabla w_{1 t}+\nabla w_{2} \nabla \tilde{w}_{2 t}
\end{array}
$$

Denoting:

$$
A_{1} \equiv\left[\begin{array}{ccc}
0 & I & 0 \\
-A_{0} & -b \mathcal{D} & -\nabla \\
0 & -d i v & -A_{D}
\end{array}\right]
$$

$A_{1}: H_{1} \rightarrow H_{1}$ with

$$
\begin{gathered}
H_{1} \equiv\left[D\left(A_{0}{ }^{1 / 2}\right]^{2} \times\left[L_{2}(\Omega)\right]^{3} \sim\left[H_{0}^{1}(\Omega)\right]^{2} \times\left[L_{2}(\Omega)\right]^{3}\right. \\
D\left(A_{1}\right)=\left\{\left(u_{1}, u_{2}, \phi\right) \in\left[D\left(A_{0}\right)\right]^{2} \times\left[D\left(A_{0}{ }^{1 / 2}\right)\right]^{2} \times D\left(A_{D}\right)\right\} \\
A_{2} \equiv\left[\begin{array}{ccc}
0 & I & 0 \\
-M^{-1} \mathcal{A} & 0 & M^{-1} A_{D} \\
0 & -A_{D} & -A_{D}
\end{array}\right]
\end{gathered}
$$

$A_{2}: H_{2} \rightarrow H_{2}$ with

$$
\begin{gathered}
H_{2} \equiv D\left(\mathcal{A}^{1 / 2}\right) \times D\left(A_{D}\right)^{1 / 2} \times L_{2}(\Omega) \sim H_{0}^{2}(\Omega) \times H_{0}^{1}(\Omega) \times L_{2}(\Omega) \\
D\left(A_{2}\right)=\left\{\left(w_{1}, w_{2}, \theta\right) \in D\left(\mathcal{A}^{1 / 2}\right) \times D\left(\mathcal{A}^{1 / 2}\right) \times D\left(A_{D}\right) ;\right. \\
\left.\mathcal{A} w_{1} \in\left[D\left(A_{D}^{1 / 2}\right)\right]^{\prime} \sim H^{-1}(\Omega)\right\}
\end{gathered}
$$

we rewrite (13) as:

$$
\frac{d}{d t}\left(\begin{array}{c}
\tilde{u}(t) \\
\tilde{u}_{t}(t) \\
\tilde{\phi}(t)
\end{array}\right)=A_{1}\left(\begin{array}{c}
\tilde{u}(t) \\
\tilde{u}_{t}(t) \\
\tilde{\phi}(t)
\end{array}\right)+\left(\begin{array}{c}
0 \\
f_{1}\left(\tilde{w}, w_{i}\right) \\
f_{3}\left(\tilde{w}, \tilde{w}_{t}, w_{i}, w_{i t}\right)
\end{array}\right)
$$




$$
\frac{d}{d t}\left(\begin{array}{c}
\tilde{w}(t) \\
\tilde{w}_{t}(t) \\
\tilde{\theta}(t)
\end{array}\right)=A_{2}\left(\begin{array}{c}
\tilde{w}(t) \\
\tilde{w}_{t}(t) \\
\tilde{\theta}(t)
\end{array}\right)+\left(\begin{array}{c}
0 \\
M^{-1} f_{2}\left(\tilde{u}, \tilde{w}, u_{i}, w_{i}\right) \\
0
\end{array}\right)
$$

It is well known that the operators $A_{1}$, (resp. $A_{2}$ ) are generators of contraction semigroup on the spaces $\left[D\left(A_{0}^{1 / 2}\right)\right]^{2} \times\left[L_{2}(\Omega)\right]^{3}$ and, respectively $D\left(\mathrm{~A}^{1 / 2}\right) \times$ $D\left(A_{D}{ }^{1 / 2}\right) \times L_{2}(\Omega)$. By standard semigroup argument we also know that these operators generate strongly continous semigroups on the dual spaces $\left[D\left(A_{1}^{*}\right)\right]^{\prime}$ and $\left[D\left(A_{2}^{*}\right)\right]^{\prime}$, respectively, where the duality is, as usual, with respect to $H_{1}, H_{2}$ topology. We apply standard energy estimates applied to (17), (18) with respect to the dual norms. This is to say, we apply the operator $A_{1}^{-1}$ [resp. $\left.A_{2}^{-1}\right]$ to both sides of equation (17) [resp. (18) ], we multiply these equations by $A_{1}^{-1}\left(\tilde{u}(t), \tilde{u}_{t}(t), \tilde{\phi}(t)\right)$ [resp. $\left.A_{2}^{-1}\left(\tilde{w}(t), \tilde{w}_{t}(t), \tilde{\theta}(t)\right)\right]$ and we integrate by parts.

This procedure produces the following negative norm estimates:

$$
\begin{gathered}
\left|\left(\tilde{u}(t), \tilde{u}_{t}(t), \tilde{\phi}(t)\right)\right|_{\left[D\left(A_{1}^{*}\right)\right]^{\prime}}^{2}+\int_{0}^{t}\left|A_{D}^{-1 / 2}(\operatorname{div} \tilde{u}+\tilde{\phi})\right|_{0, \Omega}^{2} d s \leq \\
C \int_{0}^{t}\left|\left(0, f_{1}, f_{3}\right)\right|_{\left[D\left(A_{1}^{*}\right)\right]^{\prime}}^{2} d s \\
\left.\left|\left(\tilde{w}(t), \tilde{w}_{t}(t), \tilde{\theta}(t)\right)\right|_{\left[D\left(A_{2}^{*}\right)\right]^{\prime}} \leq C \int_{0}^{t} \mid 0, M^{-1} f_{2}, 0\right)\left.\right|_{\left[D\left(A_{2}^{*}\right)\right]^{\prime}}\left|\left(\tilde{w}, \tilde{w}_{t}, \tilde{\theta}\right)\right|_{\left[D\left(A_{2}^{*}\right)\right]^{\prime}} d s
\end{gathered}
$$

On the other hand, by direct computations of $\left[A_{i}^{*}\right]^{-1}$, we obtain:

$$
\begin{gathered}
\left|\left(u_{1}, u_{2}, \phi\right)\right|_{\left[D\left(A_{1}^{*}\right)\right]^{\prime}}^{2}=\mid\left. A_{0}^{-1 / 2}\left[\nabla A_{D}^{-1}\left(\text { divu }_{1}+\phi\right)-u_{2}-b \mathcal{D} u_{1}\right]\right|_{0, \Omega} ^{2}+\left|u_{1}\right|_{0, \Omega}^{2} \\
+\left|A_{D}^{-1}\left(\operatorname{div} u_{1}+\phi\right)\right|_{0, \Omega}^{2} \\
\sim\left|u_{1}\right|_{0, \Omega}^{2}+\left|A_{0}{ }^{-1 / 2} u_{2}\right|_{0, \Omega}^{2}+\left|A_{D}^{-1} \phi\right|_{0, \Omega}^{2} \\
\left|\left(w_{1}, w_{2}, \theta\right)\right|_{\left[D\left(A_{2}^{*}\right)\right]^{\prime}}^{2}= \\
\left|\mathcal{A}^{-1 / 2}\left[M w_{2}+A_{D} w_{1}+\theta\right]\right|_{0, \Omega}^{2}+\left|M^{1 / 2} w_{1}\right|_{0, \Omega}^{2}+\left|w_{1}+A_{D}^{-1} \theta\right|_{0, \Omega}^{2} \\
\sim\left|M^{1 / 2} w_{1}\right|_{0, \Omega}^{2}+\left|\mathcal{A}^{-1 / 2} M w_{2}\right|_{0, \Omega}^{2}+\left|A_{D}^{-1} \theta\right|_{0, \Omega}^{2}
\end{gathered}
$$

Thus, in particular (after noting that $A_{D}^{-1} \operatorname{div} \in \mathcal{L}\left(\left[L_{2}(\Omega)\right] \rightarrow L_{2}(\Omega)\right.$ ) and

$$
\begin{gathered}
A_{2}^{-1}\left[0, M^{-1} f_{2}, 0\right]^{T}=-\left[\mathcal{A}^{-1} f_{2}, 0,0\right]^{T} \\
\left.|\tilde{u}(t)|_{0, \Omega}^{2}+\left|A_{0}^{-1 / 2} \tilde{u}_{t}\right|_{0, \Omega}^{2}+\mid A_{D}^{-1} \phi \tilde{(} t\right)\left.\right|_{0, \Omega} ^{2}+\int_{0}^{t}\left|A_{D}^{-1 / 2} \tilde{\phi}\right|^{2} d s \\
\leq C_{T} \int_{0}^{t}\left|A_{0}^{-1 / 2} f_{1}\right|_{0, \Omega}^{2}+\left|A_{D}^{-1} f_{3}\right|_{0, \Omega}^{2} d s \\
\left|\left(\tilde{w}, \tilde{w}_{t}, \tilde{\theta}\right)\right|_{\left[D\left(A_{2}^{*}\right)\right]^{\prime}}^{2} \leq C|\tilde{w}(t)|_{1, \Omega}^{2}+\left|\tilde{w}_{t}\right|_{0, \Omega}^{2}+\left|A_{D}^{-1} \tilde{\theta}(t)\right|_{0, \Omega}^{2} \\
\leq C \int_{0}^{t}\left|\left(\mathcal{A}^{-1 / 2} f_{2}, \mathcal{A}^{1 / 2}\left[A_{2}^{-1}\left(\tilde{w}, \tilde{w}_{t}, \tilde{\theta}\right)\right]_{1}\right)_{\Omega}\right| d s
\end{gathered}
$$


where $[\cdot]_{1}$ denotes the first coordinate of the vector in $H_{2}$.

Inequalities in (22) form a basis for the subsequent analysis.

Let $\phi \in\left[L_{2}(\Omega)\right]^{2}$. By applying boundary conditions and divergence Theorem we compute:

$$
\left(A_{0}^{-1 / 2} f_{1}, \phi\right)_{0, \Omega}=-\left(\mathcal{C}\left[f\left(\nabla w_{1}\right)-f\left(\nabla w_{2}\right)\right], \boldsymbol{\epsilon}\left(A_{0}^{-1 / 2} \phi\right)\right)_{0, \Omega}
$$

Hence,

$$
\mid\left(\left.A_{0}^{-1 / 2} f_{1}\right|_{0, \Omega} \leq C \mid\left(f\left(\nabla w_{1}\right)-\left.f\left(\nabla w_{2}\right)\right|_{0, \Omega}\right.\right.
$$

Let $P_{n}$ be the orthogonal projection on the subspace spanned by $n$ eigenvectors of $\mathcal{A}$ and let $Q_{n}=I-P_{n}$. The following "logarithmic" estimate resulting from Sobolev's embeddings and Holder's inequality is known ( eg:[43]):

$$
\begin{array}{r}
\left|\left(P_{n} f\right) g\right|_{0, \Omega} \leq C\left[\lg \left(1+\lambda_{n}\right)\right]^{1 / 2}|f|_{0, \Omega}|g|_{1, \Omega} \\
\left|\left(P_{n} f\right)\right|_{L_{\infty}(\Omega)} \leq C\left[\lg \left(1+\lambda_{n}\right)\right]^{1 / 2}|f|_{1, \Omega}
\end{array}
$$

where $\lambda_{n}$ is an eigenvalue of $\mathcal{A}$ and the constant $C$ is independent on $n$. Applying (25) and abusing slightly notation by using the projection operator applied to a vector function (meaning the projection is applied to each component) and evoking the following Sobolev's embedding:

$$
H^{\epsilon}(\Omega) \subset L_{\frac{2}{1-\epsilon}}(\Omega) ; \epsilon>0
$$

we obtain:

$$
\begin{gathered}
\mid\left(f\left(\nabla w_{1}\right)-\left.f\left(\nabla w_{2}\right)\right|_{0, \Omega} \leq C\left|\nabla \tilde{w} \times \nabla\left(w_{1}+w_{2}\right)\right|_{0, \Omega}\right. \\
\leq C\left[\left|\left(P_{n} \nabla \tilde{w}\right) \times \nabla\left(w_{1}+w_{2}\right)\right|_{0, \Omega}+\left|\left(Q_{n} \nabla \tilde{w}\right) \times \nabla\left(w_{1}+w_{2}\right)\right|_{0, \Omega}\right] \leq \\
C\left[\lg \left(1+\lambda_{n}\right)\right]^{1 / 2}|\tilde{w}|_{1, \Omega}\left[\left|w_{1}\right|_{2, \Omega}+\left|w_{2}\right|_{2, \Omega}\right]+ \\
C\left|Q_{n} \nabla \tilde{w}\right|_{\epsilon, \Omega}\left[\left|w_{1}\right|_{2, \Omega}+\left|w_{2}\right|_{2, \Omega}\right]
\end{gathered}
$$

On the other hand, by using the identification of fractional powers of eliptic operators [19], Sobolev's embeddings and Holder's inequalities we obtain:

$$
\begin{array}{r}
\left|Q_{n} \nabla \tilde{w}\right|_{\epsilon, \Omega} \leq C\left|\mathcal{A}^{1 / 4 \epsilon} Q_{n} \nabla \tilde{w}\right|_{0, \Omega} \leq C\left|\mathcal{A}^{1 / 4\left(\epsilon-\beta_{0}\right)} Q_{n}\right|_{\mathcal{L}\left(L_{2}(\Omega)\right.}|\nabla \tilde{w}|_{\beta_{0}, \Omega} \\
\leq C \lambda_{n}{ }^{1 / 4\left(\epsilon-\beta_{0}\right)}|\nabla \tilde{w}|_{\beta_{0}, \Omega} \leq C \lambda_{n}{ }^{1 / 4\left(\epsilon-\beta_{0}\right)}|\tilde{w}|_{3 / 2, \Omega}
\end{array}
$$

where $\beta_{0}<1 / 2$.

Combining ( 26), ( 27) gives:

$$
\begin{aligned}
\mid\left(\left.A_{0}^{-1 / 2} f_{1}\right|_{0, \Omega}\right. & \leq C \mid\left(f\left(\nabla w_{1}\right)-\left.f\left(\nabla w_{2}\right)\right|_{0, \Omega}\right. \\
& \leq C\left[\left[\lg \left(1+\lambda_{n}\right)\right]^{1 / 2}|\tilde{w}|_{1, \Omega}+\lambda_{n}{ }^{-\beta_{0}}\right]\left[\left|w_{1}\right|_{2, \Omega}+\left|w_{2}\right|_{2, \Omega}\right] \\
& \leq C(E(0)) \lg \left(1+\lambda_{n}\right)^{1 / 2}|\tilde{w}|_{1, \Omega}+C(E(0)) \lambda_{n}{ }^{-\beta}
\end{aligned}
$$


where $\beta<1 / 8$ and $E(0)$ denotes the initial energy of weak solutions. The estimate for $f_{2}$ is carried out next. Recalling $(21)$ with $\psi \in L_{2}(\Omega)$ we have:

$$
\begin{aligned}
\left(\mathcal{A}^{-1 / 2} f_{2}, \psi\right)_{0, \Omega}= & \left(\mathcal{C} N\left(u_{1}, w_{1}\right) \nabla w_{1}-\mathcal{C} N\left(u_{2}, w_{2}\right) \nabla w_{2}, \nabla \mathcal{A}^{-1 / 2} \psi\right)_{0, \Omega}+ \\
& \left(\nabla \tilde{w} \phi_{2}+\tilde{\phi} \nabla w_{1}, \nabla \mathcal{A}^{-1 / 2} \psi\right)_{0, \Omega}
\end{aligned}
$$

The first term in the right hand side of ( 29) can be treated exactely as in [7] to obtain

$$
\begin{gathered}
(\tilde{u}, \operatorname{divK})_{0, \Omega} \leq\left|\left(P_{n} \tilde{u}, \operatorname{divK}\right)_{0, \Omega}+\left(Q_{n} \tilde{u}, \operatorname{divK}\right)_{0, \Omega}\right| \\
\leq C\left[|\tilde{u}|_{0, \Omega}\left[\lg \left(1+\lambda_{n}\right)\right]^{1 / 2}\left|w_{1}\right|_{2, \Omega}\left|\nabla \mathcal{A}^{-1 / 2} \psi\right|_{1, \Omega}+|\tilde{u}|_{1, \Omega} \lambda_{n}{ }^{-\beta}\left|w_{1}\right|_{2, \Omega}\left|\nabla \mathcal{A}^{-1 / 2} \psi\right|_{1, \Omega}\right] \\
\leq C\left[|\tilde{u}|_{0, \Omega}\left[\lg \left(1+\lambda_{n}\right)\right]^{1 / 2}+|\tilde{u}|_{1, \Omega} \lambda_{n}{ }^{-\beta}\right]\left|w_{1}\right|_{2, \Omega}|\psi|_{0, \Omega}
\end{gathered}
$$

with

$$
K \equiv 1 / 2 \nabla w_{1} \times \nabla \mathcal{A}^{-1 / 2} \psi+\left(1 / 2 \nabla w_{1} \times \nabla \mathcal{A}^{-1 / 2} \psi\right)^{T}
$$

So, we focus our attention on the last term on the right side of (29).

$$
\begin{gathered}
\left(\phi_{2} \nabla \tilde{w}, \nabla \mathcal{A}^{-1 / 2} \psi\right)_{0, \Omega} \leq C\left|\phi_{2}\right|_{0, \Omega}\left[\left|P_{n}(\nabla \tilde{w}) \nabla \mathcal{A}^{-1 / 2} \psi\right|_{0, \Omega}+\right. \\
\left.\left|Q_{n}(\nabla \tilde{w}) \nabla \mathcal{A}^{-1 / 2} \psi\right|_{0, \Omega}\right] \\
\leq C\left|\phi_{2}\right|_{0, \Omega}\left[\left[\lg \left(1+\lambda_{n}\right)\right]^{1 / 2}|\tilde{w}|_{1, \Omega}\left|\nabla \mathcal{A}^{-1 / 2} \psi\right|_{1, \Omega}\right. \\
\left.+\left|Q_{n}(\nabla \tilde{w})\right|_{\epsilon, \Omega}\left|\nabla \mathcal{A}^{-1 / 2} \psi\right|_{1, \Omega}\right] \\
\leq C\left|\phi_{2}\right|_{0, \Omega}\left[\left[\lg \left(1+\lambda_{n}\right)\right]^{1 / 2}|\tilde{w}|_{1, \Omega}+\left|Q_{n}(\nabla \tilde{w})\right|_{\epsilon, \Omega}\right]|\psi|_{0, \Omega}
\end{gathered}
$$

Hence

$\left(\phi_{2} \nabla \tilde{w}, \nabla \mathcal{A}^{-1 / 2} \psi\right)_{0, \Omega} \leq C\left|\phi_{2}\right|_{0, \Omega}\left[\left[\lg \left(1+\lambda_{n}\right)\right]^{1 / 2}|\tilde{w}|_{1, \Omega}+\lambda_{n}^{-\beta}\left[\left|w_{1}\right|_{2, \Omega}+\left|w_{2}\right|_{2, \Omega}\right]|\psi|_{0, \Omega}\right.$

For the second part of the term $f$ we have:

$$
\begin{aligned}
\left(\tilde{\phi} \nabla w_{1}, \nabla \mathcal{A}^{-1 / 2} \psi\right)_{0, \Omega} & =\left(A_{D}^{-1 / 2} \tilde{\phi}, A_{D}^{1 / 2}\left(\nabla w_{1} \nabla \mathcal{A}^{-1 / 2} \psi\right)\right)_{0, \Omega} \\
& \leq\left|A_{D}^{-1 / 2} \tilde{\phi}\right|_{0, \Omega}\left|\nabla w_{1} \nabla \mathcal{A}^{-1 / 2} \psi\right|_{1, \Omega} \\
& \leq|\tilde{\phi}|_{-1, \Omega}\left[\left|w_{1}\right|_{2, \Omega}\left|\nabla \mathcal{A}^{-1 / 2} \psi\right|_{L_{\infty}(\Omega)}\right. \\
& \left.+\left|\nabla w_{1} D_{x} \nabla \mathcal{A}^{-1 / 2} \psi\right|_{0, \Omega}\right]
\end{aligned}
$$

where $D_{x}$ denotes a differential operator of the first order in space variable.

By using the second inequality in (25) we obtain

$$
\begin{array}{r}
\left|\nabla \mathcal{A}^{-1 / 2} \psi\right|_{L_{\infty}(\Omega)} \leq\left|P_{n} \nabla \mathcal{A}^{-1 / 2} \psi\right|_{L_{\infty}(\Omega)}+\left|Q_{n} \nabla \mathcal{A}^{-1 / 2} \psi\right|_{L_{\infty}(\Omega)} \\
\leq C\left[\ln \left(1+\lambda_{n}\right)\right]^{1 / 2}\left|\nabla \mathcal{A}^{-1 / 2} \psi\right|_{1, \Omega}+C\left|Q_{n} \nabla \mathcal{A}^{-1 / 2} \psi\right|_{1+\epsilon, \Omega} \\
\leq C\left[\ln \left(1+\lambda_{n}\right)\right]^{1 / 2}|\psi|_{0, \Omega}+C\left|\mathcal{A}^{1 / 4\left(\epsilon-\beta_{0}\right)} Q_{n}\right|_{\mathcal{L}\left(L_{2}(\Omega)\right.}\left|\nabla \mathcal{A}^{-1 / 2} \psi\right|_{1+\beta_{0}, \Omega} \\
\leq C\left[\ln \left(1+\lambda_{n}\right)\right]^{1 / 2}|\psi|_{0, \Omega}+C \lambda_{n}^{1 / 4\left(\epsilon-\beta_{0}\right)}|\psi|_{\beta_{0}, \Omega}
\end{array}
$$


By applying the first inequality in (25) we obtain:

$$
\begin{array}{r}
\left|\nabla w_{1} D_{x} \nabla \mathcal{A}^{-1 / 2} \psi\right|_{0, \Omega} \leq C\left|\nabla w_{1} P_{n} D_{x} \nabla \mathcal{A}^{-1 / 2} \psi\right|_{0, \Omega}+C\left|\nabla w_{1} Q_{n} D_{x} \nabla \mathcal{A}^{-1 / 2} \psi\right|_{0, \Omega} \\
\leq C\left[\ln \left(1+\lambda_{n}\right)\right]^{1 / 2}\left|w_{1}\right|_{2, \Omega}|\psi|_{0, \Omega}+C\left|w_{1}\right|_{2, \Omega}\left|Q_{n} D_{x} \nabla \mathcal{A}^{-1 / 2} \psi\right|_{\epsilon, \Omega} \\
\leq C\left[\ln \left(1+\lambda_{n}\right)\right]^{1 / 2}\left|w_{1}\right|_{2, \Omega}|\psi|_{0, \Omega}+C\left|w_{1}\right|_{2, \Omega}\left|\mathcal{A}^{1 / 4\left(\epsilon-\beta_{0}\right)} Q_{n}\right|_{\mathcal{L}\left(L_{2}(\Omega)\right.}\left|D_{x} \nabla \mathcal{A}^{-1 / 2} \psi\right|_{\beta_{0}, \Omega} \\
\leq C\left[\ln \left(1+\lambda_{n}\right)\right]^{1 / 2}\left|w_{1}\right|_{2, \Omega}|\psi|_{0, \Omega}+C\left|w_{1}\right|_{2, \Omega} \lambda_{n}^{1 / 4\left(\epsilon-\beta_{0}\right)}|\psi|_{\beta_{0}, \Omega}
\end{array}
$$

Combining (31)-(33) and taking $\epsilon$ suitably small yields

$$
\begin{array}{r}
\left(\tilde{\phi} \nabla w_{1}, \nabla \mathcal{A}^{-1 / 2} \psi\right)_{0, \Omega} \leq C|\psi|_{0, \Omega}\left|w_{1}\right|_{2, \Omega}\left[\ln \left(1+\lambda_{n}\right)\right]^{1 / 2}|\tilde{\phi}|_{-1, \Omega} \\
+C\left|w_{1}\right|_{2, \Omega} \lambda_{n}^{-\beta}|\tilde{\phi}|_{-1, \Omega}|\psi|_{\beta_{0}, \Omega}
\end{array}
$$

Collecting ( 29)-( 34) yields:

$$
\begin{array}{r}
\left(\mathcal{A}^{-1 / 2} f_{2}, \psi\right)_{0, \Omega} \leq C\left[\lg \left(1+\lambda_{n}\right)\right]^{1 / 2}\left[|\tilde{u}|_{0, \Omega}+|\tilde{w}|_{1, \Omega}+|\tilde{\phi}|_{-1, \Omega}\right] \\
{\left[\left|w_{1}\right|_{2, \Omega}+\left|w_{2}\right|_{2, \Omega}+\left|u_{2}\right|_{1, \Omega}+\left|u_{1}\right|_{1, \Omega}+\left|w_{1}\right|_{2, \Omega}^{2}+\left|w_{2}\right|_{2, \Omega}^{2}+\left|\phi_{1}\right|_{0, \Omega}+\left|\phi_{2}\right|_{0, \Omega}\right]|\psi|_{0, \Omega}} \\
+C \lambda_{n}{ }^{-\beta}\left[\left|w_{1}\right|_{2, \Omega}^{2}+\left|w_{2}\right|_{2, \Omega}^{2}+\left|u_{2}\right|_{1, \Omega}^{2}+\left|u_{1}\right|_{1, \Omega}^{2}+\left|\phi_{1}\right|_{0, \Omega}^{2}+\left|\phi_{2}\right|_{0, \Omega}^{2}\right]|\psi|_{0, \Omega} \\
+C\left|w_{1}\right|_{2, \Omega} \lambda_{n}^{-\beta}|\tilde{\phi}|_{-1, \Omega}|\psi|_{\beta_{0}, \Omega}
\end{array}
$$

Taking $\psi \equiv \mathcal{A}^{1 / 2}\left[A_{2}^{-1}\left(\tilde{w}, \tilde{w}_{t} . \tilde{\theta}\right)\right]_{1}$ in (35) and noting that (recall $\left.\beta_{0} \leq 1 / 2\right)$

$$
\begin{gathered}
\left|\mathcal{A}^{1 / 2}\left[A_{2}^{-1}\left(\tilde{w}, \tilde{w}_{t} \cdot \tilde{\theta}\right)\right]_{1}\right|_{\beta_{0}, \Omega} \leq C\left|\left(\tilde{w}, \tilde{w}_{t} \cdot \tilde{\theta}\right)\right|_{H_{2}} \leq C(E(0)) \\
\left|\mathcal{A}^{1 / 2}\left[A_{2}^{-1}\left(\tilde{w}, \tilde{w}_{t} \cdot \tilde{\theta}\right)\right]_{1}\right|_{0, \Omega} \leq C\left|\left(\tilde{w}, \tilde{w}_{t} \cdot \tilde{\theta}\right)\right|_{D\left(A_{2}^{*}\right)^{\prime}}
\end{gathered}
$$

yields

$$
\begin{aligned}
& \int_{0}^{t}\left|\left(\mathcal{A}^{-1 / 2} f_{2}, \mathcal{A}^{1 / 2}\left[A_{2}^{-1}\left(\tilde{w}, \tilde{w}_{t}, \tilde{\theta}\right)\right]_{1}\right)_{\Omega}\right| d s \leq \\
& \left.C(E(0)) \lg \left(1+\lambda_{n}\right)\right] \int_{0}^{t}\left[|\tilde{u}|_{0, \Omega}^{2}+|\tilde{w}|_{1, \Omega}^{2}\right] d s \\
& \quad+\lambda_{n}{ }^{-2 \beta} C_{T}(E(0))+\epsilon \int_{0}^{t}|\tilde{\phi}|_{-1, \Omega}^{2} d s+ \\
& C_{\epsilon}(E(0)) \lg \left(1+\lambda_{n}\right) \int_{0}^{t}\left|\left(\tilde{w}, \tilde{w}_{t}, \tilde{\theta}\right)\right|_{D\left(A_{2}^{*}\right)^{\prime}}^{2} d s
\end{aligned}
$$

The term $\left(f\left(\nabla w_{1}\right) \nabla w_{1}-f\left(\nabla w_{2}\right) \nabla w_{2}, \nabla \mathcal{A}^{-1 / 2} \psi\right)_{0, \Omega}$ is estimated directly as:

$$
\left(f\left(\nabla w_{1}\right) \nabla w_{1}-f\left(\nabla w_{2}\right) \nabla w_{2}, \nabla \mathcal{A}^{-1 / 2} \psi\right)_{0, \Omega} \leq C|\tilde{w}|_{1, \Omega}\left[\left|w_{1}\right|_{2, \Omega}^{2}+\left|w_{1}\right|_{2, \Omega}^{2}\right]|\psi|_{0, \Omega}
$$


Finally, we estimate the contribution of $f_{3}$ term. This is done as follows: With $\psi \in L_{2}(\Omega)$ we compute

$$
\begin{aligned}
\left(A_{D}^{-1} f_{3}, \psi\right)_{\Omega} & =\left(\nabla \tilde{w} \nabla w_{1 t}+\nabla w_{2} \nabla \tilde{w}_{t}, A_{D}^{-1} \psi\right)_{\Omega} \\
& \leq C|\nabla \tilde{w}|_{0, \Omega}\left|\nabla w_{1 t} A_{D}^{-1} \psi\right|_{0, \Omega}+\left|\tilde{w}_{t}\right|_{0, \Omega}\left|\operatorname{div}\left[\nabla w_{2} A_{D}^{-1} \psi\right]\right|_{0, \Omega} \\
& \leq C\left[|\tilde{w}|_{1, \Omega}\left|w_{1 t}\right|_{1, \Omega}+\left|\tilde{w}_{t}\right|_{0, \Omega}\left|w_{2}\right|_{2, \Omega}\right]|\psi|_{0, \Omega}
\end{aligned}
$$

Hence

$$
\left|A_{D}^{-1} f_{3}\right|_{0, \Omega} \leq C(E(0))\left[|\tilde{w}|_{1, \Omega}+\left|\tilde{w}_{t}\right|_{0, \Omega}\right]
$$

From (28), (36) and ( 38) we obtain

$$
\begin{aligned}
& \int_{0}^{t}\left[\left|\left(\mathcal{A}^{-1 / 2} f_{2}, \mathcal{A}^{1 / 2}\left[A_{2}^{-1}\left(\tilde{w}, \tilde{w}_{t}, \tilde{\theta}\right)\right]_{1}\right)_{\Omega}\right|+\left|A_{0}{ }^{-1 / 2} f_{1}\right|_{0, \Omega}^{2}+\left|A_{D}^{-1} f_{3}\right|_{0, \Omega}^{2}\right] d s \\
& \leq C(E(0))\left[\lg \left(1+\lambda_{n}\right)\right] \int_{0}^{t}\left[|\tilde{u}|_{0, \Omega}^{2}+|\tilde{w}|_{1, \Omega}^{2}+\left|\tilde{w}_{t}\right|_{0, \Omega}^{2}\right] d s+\lambda_{n}{ }^{-2 \beta} C_{T}(E(0)) \\
& +\epsilon \int_{0}^{t}|\tilde{\phi}|_{-1, \Omega}^{2} d s+C_{\epsilon}(E(0)) \lg \left(1+\lambda_{n}\right) \int_{0}^{t}\left|\left(\tilde{w} \cdot \tilde{w}_{t}, \tilde{\theta}\right)\right|_{D\left(A_{2}^{*}\right)^{\prime}}^{2} d s .
\end{aligned}
$$

and combining with ( 22 ) and Gronwall's inequality

$$
\begin{gathered}
|\tilde{u}(t)|_{0, \Omega}^{2}+|\tilde{w}(t)|_{1, \Omega}^{2}+\left|A_{D}^{-1} \tilde{\phi}(t)\right|_{0, \Omega}^{2}+\left|A_{D}^{-1} \tilde{\theta}(t)\right|_{0, \Omega}^{2} \leq \\
C_{T}(E(0)) \lambda_{n}^{-2 \beta}\left(1+\lambda_{n}\right)^{C(E(0)) t}
\end{gathered}
$$

Taking $t<T_{0}$, where $T_{0}$ is sufficiently small yields the conclusion of the Lemma for $t<T_{0}$. Applying the "boost trap" argument completes the proof of the second part of Theorem 1.1.

\subsection{Case $\gamma=0$ - proof of part 3 of Theorem 1}

The proof of the wellposedness in this case relies on the analyticity of the semigroup generated by the operator $A_{2}$ with $\gamma=0$, ie; $M=I$. Indeed, in this case we have [38] that $e^{A_{2} t}$ generates an analytic and uniformly stable semigroup on the space

$$
H_{2}=D\left(\mathcal{A}^{1 / 2}\right) \times L_{2}(\Omega) \times L_{2}(\Omega) \sim H_{0}^{2}(\Omega) \times L_{2}(\Omega) \times L_{2}(\Omega)
$$

with

$$
\begin{gathered}
D\left(A_{2}\right)=D(\mathcal{A}) \times D\left(\mathcal{A}^{1 / 2}\right) \times D\left(A_{D}\right) \sim H_{0}^{2}(\Omega) \cap H^{4}(\Omega) \times H_{0}^{2}(\Omega) \times H_{0}^{1}(\Omega) \cap H^{2}(\Omega) \\
D\left(A_{2}^{1 / 2}\right) \subset D\left(\mathcal{A}^{3 / 4}\right) \times D\left(\mathcal{A}^{1 / 4}\right) \times D\left(A_{D}^{1 / 2}\right)
\end{gathered}
$$

hence

$$
\left[D\left(A_{2}^{1 / 2}\right)\right]^{\prime} \supset\left[D\left(\mathcal{A}^{3 / 4}\right)\right]^{\prime} \times\left[D\left(\mathcal{A}^{1 / 4}\right)\right]^{\prime} \times\left[D\left(A_{D}^{1 / 2}\right)\right]^{\prime}
$$




\section{STEP 1:}

By the same Galerkin method as introduced in the first subsection, we obtain the existence of finite energy solutions which obey the estimate:

$$
E(t)+2 \int_{0}^{t}\left[|\nabla \phi|_{0, \Omega}^{2}+|\nabla \theta|_{0, \Omega}^{2}+\left|\operatorname{curl} q_{t}\right|_{0, \Omega}^{2}\right] d t=E(0)
$$

where we recall, $E(t)=E_{0}(t)$, taking $\gamma=0$. In fact, the argument is identical as before with the only difference that in order to pass with the limit on nonlinear terms in the equation for $\phi$ we need to write $\nabla w_{h} \nabla w_{h, t}=1 / 2 \frac{d}{d t}\left|\nabla w_{h}\right|^{2}$ and use test functions which are regular in time.

(41) and inequality in (7) give us the apriori regularity of the solutions:

$$
\begin{array}{r}
u \in C\left(0, T ;\left[H_{0}^{1}(\Omega)\right]^{2}\right) \cap C^{1}\left(0, T ;\left[L_{2}(\Omega)\right]^{2}\right) \\
w \in C\left(0, T ;\left[H_{0}^{2}(\Omega)\right]^{2}\right) \cap C^{1}\left(0, T ;\left[L_{2}(\Omega)\right]^{2}\right) \\
(\phi, \theta) \in L_{2}\left(0, T ;\left[H_{0}^{1}(\Omega)\right]^{2}\right) \cap C\left(0, T ;\left[L_{2}(\Omega)\right]^{2}\right)
\end{array}
$$

Our main point is to show that the variable $w$ displays more regularity.

STEP 2: Let $H_{1} \times H_{2}=\left[H_{0}^{1}(\Omega)\right]^{2} \times\left[L_{2}(\Omega)\right]^{2} \times L_{2}(\Omega) \times H_{0}^{2}(\Omega) \times L_{2}(\Omega) \times L_{2}(\Omega)$

Lemma 2.1 Let $\gamma=0$ and the initial data be of finite energy i.e.:

$\left(u_{0}, u_{1}, \phi_{0}, w_{0}, w_{1}, \theta_{0}\right) \in H_{1} \times H_{2}$ then

$$
\left(w, w_{t}\right) \in L_{2}\left(0, T ; H^{3}(\Omega) \times H^{1}(\Omega)\right) .
$$

for all $T>0$.

where $H_{1} \times H_{2}=\left[H_{0}^{1}(\Omega)\right]^{2} \times\left[L_{2}(\Omega)\right]^{2} \times L_{2}(\Omega) \times H_{0}^{2}(\Omega) \times L_{2}(\Omega) \times L_{2}(\Omega)$

\section{Proof:}

We start by writing the solution to the plate component via semigroup formula:

$$
\left(\begin{array}{c}
w(t) \\
w_{t}(t) \\
\theta(t)
\end{array}\right)=e^{A_{2} t}\left(\begin{array}{c}
w_{0} \\
w_{1} \\
\theta_{0}
\end{array}\right)+\int_{0}^{t} e^{A_{2}(t-s)}\left(\begin{array}{c}
0 \\
\operatorname{div}[\mathcal{C} N(u, w) \nabla(w)+\phi \nabla w] \\
0
\end{array}\right) d t
$$

Hence by (40) and the regularity $\mathcal{A}^{-1 / 4} \operatorname{div} \in \mathcal{L}\left(L_{2}(\Omega)\right)$

$$
\begin{array}{r}
\left|A_{2}^{-1 / 2}\left(\begin{array}{c}
0 \\
\operatorname{div}[\mathcal{C} N(u, w) \nabla(w)+\phi \nabla w] \\
0
\end{array}\right)\right|_{H_{2}} \\
\leq C\left|\mathcal{A}^{-1 / 4} \operatorname{div}[\mathcal{C} N(u, w) \nabla(w)+\phi \nabla w]\right|_{0, \Omega} \\
\leq C|N(u, w) \nabla(w)+\phi \nabla w|_{0, \Omega} \\
\leq C\left[|N(u, w)|_{0, \Omega}+|\phi|_{0, \Omega}\right]|\nabla w|_{L_{\infty}(\Omega)}
\end{array}
$$

Going back to the semigroup formula, we obtain:

$$
A_{2}^{1 / 2}\left(\begin{array}{c}
w(t) \\
w_{t}(t) \\
\theta(t)
\end{array}\right)=A_{2}^{1 / 2} e^{A_{2} t}\left(\begin{array}{c}
w_{0} \\
w_{1} \\
\theta_{0}
\end{array}\right)+
$$




$$
+\int_{0}^{t} A_{2} e^{A_{2}(t-s)} A_{2}^{-1 / 2}\left(\begin{array}{c}
0 \\
\operatorname{div}[\mathcal{C} N(u, w) \nabla(w)+\phi \nabla w] \\
0
\end{array}\right) d t
$$

Singular estimates available for analytic, contractive and stable semigroups [10] yield:

$$
\left|A_{2}^{1 / 2} e^{A_{2} t} x\right|_{L_{2}\left(0, T ; H_{2}\right)} \leq C|x|_{H_{2}} ; \quad\left|\int_{0}^{t} A_{2} e^{A_{2}(t-s)} f(s) d s\right|_{L_{2}\left(0, T ; H_{2}\right)} \leq C|f|_{L_{2}\left(0, T ; H_{2}\right)}
$$

So

$$
\begin{aligned}
\left.\left|A_{2}^{1 / 2}\left(\begin{array}{c}
w(t) \\
w_{t}(t) \\
\theta(t)
\end{array}\right)\right|\right|_{L_{2}\left(0, T ; H_{2}\right)} & \leq C\left|\left(\begin{array}{c}
w_{0} \\
w_{1} \\
\theta_{0}
\end{array}\right)\right|_{H_{2}}+\mid\left[|N(u, w)|_{0, \Omega}\right. \\
& \left.+|\phi|_{0, \Omega}\right]\left.|\nabla w|_{L_{\infty}(\Omega)}\right|_{L_{2}(0, T)} \\
& \leq C E(0)^{1 / 2}+C E(0)^{1 / 2}|w|_{L_{2}\left(0, T ; H^{2+\epsilon}(\Omega)\right)}
\end{aligned}
$$

where we have used the a priori bound (41) and Sobolev's embedding $H^{1+\epsilon}(\Omega) \subset$ $L_{\infty}(\Omega)$. From (46) and applying moment and interpolation inequalities we conclude the estimate

$$
\begin{array}{r}
\int_{0}^{T}\left[|w(t)|_{3, \Omega}^{2}+\left|w_{t}(t)\right|_{1, \Omega}^{2} d t \leq C E(0)+C E(0) \int_{0}^{T}|w(t)|_{2+\epsilon, \Omega}^{2} d t\right. \\
\leq C E(0)+C_{\epsilon} E(0)^{2} \int_{0}^{T}|w(t)|_{2, \Omega}^{2} d t+\epsilon \int_{0}^{T}|w(t)|_{3, \Omega}^{2} d t
\end{array}
$$

Taking $\epsilon$ suitably small yields

$$
\int_{0}^{T}\left[|w(t)|_{3, \Omega}^{2}+\left|w_{t}(t)\right|_{1, \Omega}^{2}\right] d t \leq C E(0)+C E(0)^{2} \int_{0}^{T}|w(t)|_{2, \Omega}^{2} d t \leq C_{T} E(0)
$$

which gives the result of the Lemma 3. $\square$.

STEP 3:

Using the improved regularity of the variable $w$ we shall prove the uniqueness of finite energy solutions. To this end we go back to the equations (17) and (18) with $M=I$. Functions $f_{1}, f_{2}$ and $f_{3}$ are the same as in (14). Standard energy method gives the following inequality

$$
\left|\begin{array}{c}
\tilde{u}(t) \\
\tilde{u}_{t}(t) \\
\tilde{\phi}(t)
\end{array}\right|_{H_{1}}+\left[\int_{0}^{t}|\nabla \phi|_{0, \Omega}^{2} d s\right]^{1 / 2} \leq C \int_{0}^{t}\left|f_{1}\right|_{0, \Omega} d s+C\left[\int_{0}^{t}\left|A_{D}^{-1 / 2} f_{3}\right|_{0, \Omega}^{2} d s\right]^{1 / 2}
$$

The appropriate norms of $f_{i}$ are estimated below:

$$
\begin{array}{r}
\left|f_{1}\left(\tilde{w}, w_{i}\right)\right|_{0, \Omega} \leq C\left|\nabla \tilde{w} \times \nabla\left(w_{1}+w_{2}\right)\right|_{1, \Omega} \leq C|\tilde{w}|_{2, \Omega}\left|\nabla\left(w_{1}+w_{2}\right)\right|_{L_{\infty}(\Omega)} \\
+|\nabla \tilde{w}|_{L_{p}(\Omega)}\left|\left(w_{1}+w_{2}\right)\right|_{W^{2, \bar{p}}(\Omega)} \leq C|\tilde{w}|_{2, \Omega}\left[\left|\nabla\left(w_{1}+w_{2}\right)\right|_{L_{\infty}(\Omega)}+\left|\left(w_{1}+w_{2}\right)\right|_{W^{2, \bar{p}}(\Omega)}\right] \\
\leq C|\tilde{w}|_{2, \Omega}\left|\left(w_{1}+w_{2}\right)\right|_{3, \Omega}
\end{array}
$$


Similarily as in (45) we also obtain

$$
\left|A_{2}^{-1 / 2-\rho}\left(\begin{array}{c}
0 \\
f_{2}\left(\tilde{u}, \tilde{w}, u_{i}, w_{i}\right) \\
0
\end{array}\right)\right|_{H_{2}} \leq C(E(0))\left[|\tilde{u}|_{1, \Omega}+|\tilde{w}|_{2, \Omega}+|\tilde{\phi}|_{0, \Omega}\right]
$$

where $\rho$ can be taken arbitrarily small. To compute $\left|A_{D}^{-1 / 2} f_{3}\right|_{0, \Omega}$ we evaluate the $L_{2}$ inner product of $A_{D}^{-1 / 2} f_{3}$ with an arbitrary $L_{2}$ function $\psi$. Relevant computations are given below.

$$
\begin{gathered}
\left(A_{D}^{-1 / 2} \nabla w_{2} \nabla \tilde{w}_{t}, \psi\right)_{\Omega}=-\left(\tilde{w}_{t}, \operatorname{div}\left[\nabla w_{2} A_{D}^{-1 / 2} \psi\right]\right)_{\Omega} \\
\leq C\left|\tilde{w}_{t}\right|_{0, \Omega}\left|w_{2}\right|_{2+\epsilon, \Omega}|\psi|_{0, \Omega} \\
\left.\left(A_{D}^{-1 / 2} \nabla w_{1, t} \nabla \tilde{w}, \psi\right)_{\Omega}=\left(\nabla w_{1, t}, \nabla \tilde{w} A_{D}^{-1 / 2} \psi\right]\right)_{\Omega} \leq C\left|w_{1, t}\right|_{1-\epsilon, \Omega}\left|\nabla \tilde{w} A_{D}^{-1 / 2} \psi\right|_{\epsilon, \Omega} \\
\leq C\left|w_{1, t}\right|_{1-\epsilon, \Omega}|\tilde{w}|_{1+2 \epsilon, \Omega}|\psi|_{0, \Omega}
\end{gathered}
$$

Hence

$$
\begin{array}{r}
\left|A_{D}^{-1 / 2} f_{3}\left(\tilde{w}, \tilde{w}_{t}\right)\right|_{0, \Omega} \leq C\left[\left|\tilde{w}_{t}\right|_{0, \Omega}\left|w_{2}\right|_{2+\epsilon, \Omega}+|\tilde{w}|_{2, \Omega}\left|w_{1, t}\right|_{1-\epsilon, \Omega}\right] \\
\leq \epsilon\left[\left|\tilde{w}_{t}\right|_{0, \Omega}|w|_{3, \Omega}+|\tilde{w}|_{2, \Omega}\left|\nabla w_{t}\right|_{0, \Omega}\right]+C_{\epsilon}\left[\left|\tilde{w}_{t}\right|_{0, \Omega}|w|_{2, \Omega}+|\tilde{w}|_{2, \Omega}\left|w_{t}\right|_{0, \Omega}\right]
\end{array}
$$

Now, by Lemma 3, (48), (49), (53) we obtain

$$
\begin{array}{r}
\left|\begin{array}{c}
\tilde{u}(t) \\
\tilde{u}_{t}(t) \\
\tilde{\phi}(t)
\end{array}\right|_{H_{1}} \leq C \int_{0}^{t}|\tilde{w}|_{2, \Omega}\left|w_{1}+w_{2}\right|_{3, \Omega} d t+\left[\int_{0}^{t}\left|A_{D}^{-1 / 2} f_{3}\right|_{0, \Omega}^{2} d s\right]^{1 / 2} \\
\leq C\left[\int_{0}^{t}|\tilde{w}|_{2, \Omega}^{2} d s\right]^{1 / 2}\left[\int_{0}^{t}\left|w_{1}+w_{2}\right|_{3, \Omega}^{2} d s\right]^{1 / 2} \\
+\epsilon\left[\left|\tilde{w}_{t}\right|_{L_{\infty}\left(0, t, L_{2}(\Omega)\right.}+|\tilde{w}|_{L_{\infty}\left(0, t, H^{2}(\Omega)\right.}\right]\left[\int_{0}^{t}\left|w_{2}\right|_{3, \Omega}^{2}+\left|w_{1, t}\right|_{1, \Omega}^{2} d s\right]^{1 / 2}+ \\
C_{\epsilon}(E(0))\left[\int_{0}^{t}|\tilde{w}|_{2, \Omega}^{2}+\left|\tilde{w}_{t}\right|_{0, \Omega}^{2} d s\right]^{1 / 2} \\
\leq C_{\epsilon}(E(0))\left[\int_{0}^{t}|\tilde{w}(s)|_{2, \Omega}^{2} d s+\int_{0}^{t}\left|\tilde{w}_{t}(s)\right|_{0, \Omega}^{2} d s\right]^{1 / 2} \\
+\epsilon C(E(0))\left[\left|\tilde{w}_{t}\right|_{L_{\infty}\left(0, t, L_{2}(\Omega)\right.}+|\tilde{w}|_{L_{\infty}\left(0, t, H^{2}(\Omega)\right.}\right]
\end{array}
$$

On the other hand, by the analyticity of $e^{A_{2} t}$ and (50)

$$
\begin{array}{r}
\left|\begin{array}{c}
\tilde{w}(t) \\
\tilde{w}_{t}(t) \\
\tilde{\theta}(t)
\end{array}\right|_{H_{2}} \leq\left|\int_{0}^{t} A_{2}^{1 / 2+\rho} e^{A_{2}(t-s)} A_{2}^{-1 / 2-\rho}\left(\begin{array}{c}
0 \\
f_{2}\left(\tilde{u}, \tilde{w}, u_{i}, w_{i}\right) \\
0
\end{array}\right) d s\right|_{H_{2}} \\
\leq C(E(0)) \int_{0}^{t} \frac{1}{(t-s)^{1 / 2+\rho}}\left[|\tilde{u}(s)|_{1, \Omega}+|\tilde{w}(s)|_{2, \Omega}+|\tilde{\phi}|_{0, \Omega}\right] d s \\
\leq C(E(0))\left[\int_{0}^{t} \frac{1}{(t-s)^{1 / 2+\rho}} d s\right]^{1 / 2} . \\
{\left[\int_{0}^{t} \frac{1}{(t-s)^{1 / 2+\rho}}\left[|\tilde{u}(s)|_{1, \Omega}^{2}+|\tilde{w}(s)|_{2, \Omega}^{2}+|\phi|_{0, \Omega}^{2}\right] d s\right]^{1 / 2}}
\end{array}
$$


From (54), (55) and taking $\epsilon$ suitably small yields

$$
\begin{aligned}
& |\tilde{u}(t)|_{1, \Omega}^{2}+|\tilde{\phi}(t)|_{0, \Omega}^{2}+|\tilde{w}(t)|_{2, \Omega}^{2}+\left|\tilde{w}_{t}(t)\right|_{0, \Omega}^{2}+|\tilde{\theta}(t)|_{0, \Omega}^{2} \\
\leq & C_{T}(E(0)) \cdot \\
& \int_{0}^{t} \frac{1}{(t-s)^{1 / 2+\rho}}\left[|\tilde{u}(s)|_{1, \Omega}^{2}+|\tilde{w}(s)|_{2, \Omega}^{2}+\left|\tilde{w}_{t}(s)\right|_{0, \Omega}^{2}+|\tilde{\phi}|_{0, \Omega}^{2} x\right] d s
\end{aligned}
$$

This last inequality and Cronwall's inequality with $L_{1}$ kernel yield:

$$
\tilde{u}=\tilde{w}=\tilde{\phi}=\tilde{\theta} \equiv 0
$$

as desired for the final conclusion of part 3 of Theorem 1.1. $\square$

\section{Proof of Theorem 2}

\subsection{Preliminaries}

The following results are straightforward counterparts of Lemma 2.1 and Lemma 2.2 proved in [7].

Lemma 3.1 Let $u, w$ be a finite energy solution of system (1). Then, for any $s \leq t$

$$
E_{\gamma}(t)+2 \int_{s}^{t} \int_{\Omega}\left[\left.\nabla \theta\right|^{2}+|\nabla \phi|^{2}+b\left|c u r l q_{t}\right|^{2} d \Omega d t=E_{\gamma}(s)\right.
$$

The following notation will be used throughout.

$$
Q \equiv[0, T] \times \Omega, \Sigma \equiv[0, T] \times \Gamma
$$

Lemma 3.2 Let $u, w, \theta, \phi$ be a finite energy solution corresponding to (1),(2). Then, there exists a constant $C_{T}$ such that the following trace regularity takes place.

$$
\begin{gathered}
\int_{\Sigma}\left[\left|\frac{\partial}{\partial \nu} u\right|^{2}\right. \\
\left.+|\Delta w|^{2}\right] d \Sigma \leq C_{T}\left[E_{\gamma}(0)+E_{\gamma}(0) \int_{0}^{T}\left[|\theta|_{1, \Omega}^{2}+|\phi|_{1, \Omega}^{2}\right] d t\right]
\end{gathered}
$$

Remark 3.3 Notice that the regularity of the trace of $\frac{\partial}{\partial \nu} u, \Delta w$ proclaimed by the Lemma 3.2, does not follow from the standard interior regularity of finite energy solutions via the Trace Theory. These are independent regularity results. 


\subsection{Exponential Decay Rates}

\subsubsection{Main Estimate}

The main aim in this section is to prove the following stabilizability estimate

Lemma 3.4 Let $u, w, \theta, \phi$ be a regular solution to (1), (2). Then, there exists $T$ large enough such that

$E_{\gamma}(0)+E_{\gamma}(T)+\int_{0}^{T} E_{\gamma}(t) d t \leq C_{\gamma, T}\left(E_{\gamma}(0)\right) \int_{\Omega}\left[|\nabla \theta|_{0, \Omega}^{2}+|\nabla \phi|_{0, \Omega}^{2}+b\left|c u r l q_{t}\right|_{0, \Omega}^{2}\right] d t$

where $C_{\gamma, T} \equiv \frac{1}{\gamma} C_{T}$ when $\gamma>0$ and $C_{\gamma, T} \equiv C_{T}$ when $\gamma=0$.

The estimate of the Lemma above, critical to the proof of the main stabilliz ability result, is an inverse type of the estimate. Indeed, it allows to reconstruct the energy of the system from the measurments of thermal components and solenoidal part of the velocity $u_{t}$. The reminder of this section is devoted to the proof of the Lemma 3.4. Here, the strategy used for the proof is to apply the multipliers used in the context of thermoelastic plates in [3] (see also [4, 5]) and thermoelastic systems [9].

Remark 3.5 The result of Lemma 5 can be extended to hold for all finite energy (weak) solutions. Indeed, this can be accomplished by applying a special "regularization" argument as in [28]. In order to avoid the additional technical complications and for the sake of clarity of exposition we shall not do this here and for details we refer the reader to [28] and also [20], [21].

\subsubsection{First Estimate}

In this subsection we shall prove a preliminary estimate which gives the estimate for the kinetic energy modulo "small" contribution of the potential energy and the dissipative terms.

Lemma 3.6 Let $u, w, \theta, \phi$ be a regular solution to (1), (2). Then, for any $\epsilon>0$, $T>0$ the following estimate takes place:

$$
\begin{gathered}
\int_{0}^{T} E_{k, \gamma}(t) d t \leq \epsilon \int_{0}^{T} E_{p}(t) d t+ \\
C_{\gamma, \epsilon, T}\left(E_{\gamma}(0)\right) \int_{0}^{T}\left[|\theta|_{1, \Omega}^{2}+|\phi|_{1, \Omega}^{2}+\left|\operatorname{curl} q_{t}\right|_{0, \Omega}^{2}\right] d t+C_{\gamma} E_{\gamma}(0)
\end{gathered}
$$

where $C_{\gamma, \epsilon, T} \equiv \frac{1}{\gamma} C_{\epsilon, T}$ when $\gamma>0$ and $C_{\gamma, \epsilon, T} \equiv C_{\epsilon, T}$ when $\gamma=0$.

\section{proof}

STEP 1 
Let $A_{D}$ denotes negative Laplasjan with the zero Dirichlet boundary data. We apply the variational form (11) with the following choice of test functions:

$$
\xi \equiv \nabla A_{D}^{-1} \phi ; \quad \psi \equiv A_{D}^{-1} \theta
$$

Relevant computations are provided below.

An application of the first multiplier and integration by parts gives:

$$
\begin{array}{r}
\int_{0}^{T}\left(u_{t t}, \nabla A_{D}^{-1} \phi\right)_{\Omega} d t=\left.\left(u_{t}, \nabla A_{D}^{-1} \phi\right)_{\Omega}\right|_{0} ^{T}-\int_{0}^{T}\left(u_{t}, \nabla A_{D}^{-1} \phi_{t}\right)_{\Omega} d t \\
\text { recalling equation for } \phi \\
=\left.\left(u_{t}, \nabla A_{D}^{-1} \phi\right)_{\Omega}\right|_{0} ^{T}-\int_{0}^{T}\left(u_{t}, \nabla A_{D}^{-1}\left(-A_{D}\right) \phi-\nabla A_{D}^{-1}\left(d i v u_{t}-\nabla w \nabla w_{t}\right)\right)_{\Omega} d t \\
=\left.\left(u_{t}, \nabla A_{D}^{-1} \phi\right)_{\Omega}\right|_{0} ^{T}+\int_{0}^{T}\left(u_{t}, \nabla \phi-\nabla A_{D}^{-1} A_{D} p_{t}+\nabla A_{D}^{-1} \nabla w \nabla w_{t}\right)_{\Omega} d t \\
=\left.\left(u_{t}, \nabla A_{D}^{-1} \phi\right)_{\Omega}\right|_{0} ^{T}+ \\
\int_{0}^{T}\left(\nabla p_{t}+\operatorname{curl} q_{t}, \nabla \phi-\nabla p_{t}+\nabla A_{D}^{-1} \nabla w \nabla w_{t}\right)_{\Omega} d t=\left.\left(u_{t}, \nabla A_{D}^{-1} \phi\right)_{\Omega}\right|_{0} ^{T} \\
+\int_{0}^{T}\left(\nabla p_{t}, \nabla \phi-\nabla p_{t}+\nabla A_{D}^{-1} \nabla w \nabla w_{t}\right)_{\Omega} d t
\end{array}
$$

On the other hand, taking an arbitrary $\psi \in L_{2}(\Omega)$ and $p>2$

$$
\begin{array}{r}
\left(\nabla A_{D}^{-1} \nabla w \nabla w_{t}, \psi\right)_{\Omega}=-\left(\nabla w_{t}, \nabla w A_{D}^{-1} \operatorname{div} \psi\right)_{\Omega}=\left(w_{t}, \operatorname{div}\left[\nabla w A_{D}^{-1} \operatorname{div} \psi\right]\right)_{\Omega} \leq \\
\left.C\left|w_{t}\right|_{L_{p}(\Omega)} \mid \nabla w A_{D}^{-1} \operatorname{div} \psi\right]\left.\right|_{W^{1, \bar{p}}(\Omega)} \leq C\left|w_{t}\right|_{\epsilon, \Omega}|w|_{2, \Omega}|\psi|_{0, \Omega}
\end{array}
$$

Hence

$$
\int_{0}^{T}\left|\nabla A_{D}^{-1} \nabla w \nabla w_{t}\right|_{0, \Omega}^{2} d t \leq C \int_{0}^{T}\left|w_{t}\right|_{\epsilon, \Omega}^{2}|w|_{2, \Omega}^{2} d t \leq C E_{\gamma}(0) \int_{0}^{T}\left|w_{t}\right|_{\epsilon_{0}, \Omega}^{2} d t
$$

From (57) and (59) we obtain:

$$
\begin{aligned}
\int_{0}^{T}\left(u_{t t}, \nabla A_{D}^{-1} \phi\right)_{\Omega} d t \leq- & \int_{0}^{T}\left|\nabla p_{t}\right|_{0, \Omega}^{2} d t+C E_{\gamma}(0)+\epsilon \int_{0}^{T}\left|\nabla p_{t}\right|_{0, \Omega}^{2} d t \\
& +C_{\epsilon} \int_{0}^{T}|\nabla \phi|_{0, \Omega}^{2} d t+C E_{\gamma}(0) \int_{0}^{T}\left|w_{t}\right|_{\epsilon_{0}, \Omega}^{2} d t
\end{aligned}
$$

When $\gamma>0$ and taking $\epsilon<1 / 2(60)$ implies

$$
\begin{array}{r}
\int_{0}^{T}\left(u_{t t}, \nabla A_{D}^{-1} \phi\right)_{\Omega} d t \leq-1 / 2 \int_{0}^{T}\left|\nabla p_{t}\right|_{0, \Omega}^{2} d t+C E_{\gamma}(0)+C \int_{0}^{T}|\nabla \phi|_{0, \Omega}^{2} d t \\
+\frac{C}{\gamma} E_{\gamma}(0) \int_{0}^{T} \gamma\left|w_{t}\right|_{1, \Omega}^{2} d t
\end{array}
$$

When $\gamma=0$ we use instead the inequality in Lemma 4.2 which via interpolation leads to 


$$
\int_{0}^{T}\left|w_{t}\right|_{\epsilon_{0}, \Omega}^{2} d t \leq C E(0)+\epsilon_{0} \int_{0}^{T}|w|_{2, \Omega}^{2}+C_{\epsilon}(E(0)) \int_{0}^{T}\left|w_{t}\right|_{0, \Omega}^{2}
$$

Combining the above with (60) gives

$$
\begin{array}{r}
\int_{0}^{T}\left(u_{t t}, \nabla A_{D}^{-1} \phi\right)_{\Omega} d t \leq-1 / 2 \int_{0}^{T}\left|\nabla p_{t}\right|_{0, \Omega}^{2} d t+C E(0)+C \int_{0}^{T}|\nabla \phi|_{0, \Omega}^{2} d t \\
+\epsilon_{0} \int_{0}^{T} E_{p}(t) d t+C_{\epsilon_{0}}(E(0)) \int_{0}^{T}\left|w_{t}\right|_{0, \Omega}^{2} d t
\end{array}
$$

In addition, the following relations will be used.

$$
\begin{array}{r}
\mid\left(\mathcal{C}[\boldsymbol{\epsilon}(u)+f(\nabla w)], \boldsymbol{\epsilon}\left(\nabla A_{D}^{-1} \phi\right)_{\Omega}-\left.\left\langle\mathcal{C} \boldsymbol{\epsilon}(u) \nu, \nabla A_{D}^{-1} \phi\right\rangle_{\Gamma}|\leq \epsilon| N(u, w)\right|_{0, \Omega} ^{2}+\right. \\
C_{\epsilon, \epsilon_{1}}|\phi|_{0, \Omega}^{2}+\epsilon_{1}\left|\frac{\partial}{\partial \nu} u\right|_{0, \Gamma}^{2}
\end{array}
$$

$$
\left|\left(\nabla \phi, \nabla A_{D}^{-1} \phi\right)_{\Omega}\right| \leq C|\phi|_{1, \Omega}^{2} ; \quad\left(\operatorname{curl} u_{t}, \nabla A_{D}^{-1} \phi\right)_{\Omega}=0
$$

Combining (60) [resp. (62)] with (63),(64) and with the variational form (11), after recalling $u=\nabla p+$ curl $q$ leads to the following inequalities

$$
\begin{aligned}
\int_{0}^{T}\left|u_{t}\right|_{0, \Omega}^{2} d t \leq & C E_{\gamma}(0)+\frac{C}{\gamma} E_{\gamma}(0) \int_{0}^{T} \gamma\left|\nabla w_{t}\right|_{0, \Omega}^{2} d t+\epsilon \int_{0}^{T} E_{p}(t) d t \\
& +\epsilon_{1} \int_{0}^{T}\left|\frac{\partial}{\partial \nu} u\right|_{0, \Gamma}^{2} d t+C_{\epsilon, \epsilon_{1}} \int_{0}^{T}\left[|\phi|_{1, \Omega}^{2}+\mid \text { curl }\left._{t}\right|_{0, \Omega} ^{2}\right] d t
\end{aligned}
$$

and when $\gamma=0$

$$
\begin{array}{r}
\int_{0}^{T}\left|u_{t}\right|_{0, \Omega}^{2} d t \leq C E_{\gamma}(0)+C E(0) \int_{0}^{T}\left|w_{t}\right|_{0, \Omega}^{2} d t+\epsilon \int_{0}^{T} E_{p}(t) d t \\
+\epsilon_{1} \int_{0}^{T}\left|\frac{\partial}{\partial \nu} u\right|_{0, \Gamma}^{2} d t+C_{\epsilon, \epsilon_{1}} \int_{0}^{T}\left[|\phi|_{1, \Omega}^{2}+\mid \text { curl }\left._{t}\right|_{0, \Omega} ^{2}\right] d t
\end{array}
$$

Similarily, application of the second multiplier $A_{D}^{-1}$ and the use of thermal equation for $\theta$ followed by integration by parts give: (see [3] for detailed calculations)

$$
\begin{array}{r}
\int_{0}^{T}\left(w_{t t}-\gamma \Delta w_{t t}, A_{D}^{-1} \theta\right)_{\Omega} d t+\int_{0}^{T}\left[\left|w_{t}\right|_{0, \Omega}^{2}+\gamma\left|\nabla w_{t}\right|_{0, \Omega}^{2}\right] d t \\
\leq C \int_{0}^{T}|\theta|_{1, \Omega}^{2} d t+C E_{\gamma}(0) \\
\int_{0}^{T}\left[\left(\Delta w, \Delta A_{D}^{-1} \theta\right)_{\Omega}-\left\langle\Delta w, \frac{\partial}{\partial \nu} A_{D}^{-1} \theta\right\rangle_{\Gamma}\right] d t \\
\leq \epsilon \int_{0}^{T}|\Delta w|_{0, \Omega}^{2} d t+\epsilon_{1} \int_{\Sigma}|\Delta w|^{2} d \Sigma+C_{\epsilon, \epsilon_{1}} \int_{0}^{T}|\theta|_{0, \Omega}^{2} d t
\end{array}
$$




$$
\begin{gathered}
\left|\left(\mathcal{C}[\boldsymbol{\epsilon}(u)+f(\nabla w)], \nabla A_{D}^{-1} \theta \times \nabla w\right)_{\Omega}\right| \leq \epsilon|N(u, w)|_{0, \Omega}^{2}+C_{\epsilon}\left|\nabla w \nabla A_{D}^{-1} \theta\right|_{0, \Omega}^{2} \\
\leq \epsilon|N(u, w)|_{0, \Omega}^{2}+C_{\epsilon}|\nabla w|_{L_{4}(\Omega)}^{2}\left|\nabla A_{D}^{-1} \theta\right|_{L_{4}(\Omega)}^{2} \\
\left(\phi \nabla w, \nabla A_{D}^{-1} \theta\right)_{\Omega} \leq C|w|_{1, \Omega}|\phi|_{1, \Omega}|\theta|_{1, \Omega}
\end{gathered}
$$

Hence

$$
\int_{0}^{T}\left(\phi \nabla w, \nabla A_{D}^{-1} \theta\right)_{\Omega} d t \leq C\left(E_{\gamma}(0)\right) \int_{0}^{T}|\phi|_{1, \Omega}^{2}+|\theta|_{1, \Omega}^{2} d t
$$

By Sobolev's embeddings and dissipation equality in lemma 7.

$$
\begin{aligned}
\left|\int_{0}^{T}\left(\mathcal{C}[\boldsymbol{\epsilon}(u)+f(\nabla w)], \nabla A_{D}^{-1} \theta \times \nabla w\right)_{\Omega} d t\right| & \leq \epsilon \int_{0}^{T}|N(u, w)|_{0, \Omega}^{2} d t+ \\
C_{\epsilon} E_{\gamma}(0) \int_{0}^{T}\left|\nabla A_{D}^{-1} \theta\right|_{L_{4}(\Omega)}^{2} d t & \leq \epsilon \int_{0}^{T}|N(u, w)|_{0, \Omega}^{2} d t \\
& +C_{\epsilon} E_{\gamma}(0) \int_{0}^{T}|\theta|_{0, \Omega}^{2} d t
\end{aligned}
$$

From (67)-(72) we infer

$$
\begin{aligned}
\int_{0}^{T}\left|w_{t}\right|_{0, \Omega}^{2}+\gamma\left|\nabla w_{t}\right|_{0, \Omega}^{2} d t & \leq C E_{\gamma}(0)+\epsilon \int_{0}^{T} E_{p}(t) d t \\
& +\epsilon_{1} \int_{\Sigma}\left[\left|\frac{\partial}{\partial \nu} u\right|^{2}+|\Delta w|^{2}\right] d \Sigma \\
& +C_{\epsilon, \epsilon_{1}} E_{\gamma}(0) \int_{0}^{T}\left[|\phi|_{1, \Omega}^{2}+|\theta|_{1, \Omega}^{2}+\left|\operatorname{curl} q_{t}\right|_{0, \Omega}^{2}\right] d t
\end{aligned}
$$

We multiply both sides of (73) by a suitably large constant (propotional to $\frac{1}{\gamma}$ in the case $\gamma>0$ ) and we add the result to the inequalities in (65) (when $\gamma>0$ ) and (66) (when $\gamma=0$ ) to obtain

$$
\begin{array}{r}
\int_{0}^{T} E_{k, \gamma} d t \leq \epsilon \int_{0}^{T} E_{p}(t) d t+C_{\gamma, \epsilon, \epsilon_{1}} E_{\gamma}(0) \int_{0}^{T}\left[|\phi|_{1, \Omega}^{2}+|\theta|_{1, \Omega}^{2}+\left|\operatorname{curl} q_{t}\right|_{0, \Omega}^{2}\right] d t+ \\
C_{\gamma} E_{\gamma}(0)+\epsilon_{1} \int_{\Sigma}\left[\left|\frac{\partial}{\partial \nu} u\right|^{2}+|\Delta w|^{2}\right] d \Sigma
\end{array}
$$

\section{STEP 2}

Applying trace estimate given by Lemma 3.2 to inequality in (74) yields:

$$
\begin{array}{r}
\int_{0}^{T} E_{k, \gamma} d t \leq \epsilon \int_{0}^{T} E_{p}(t) d t+C_{\gamma, \epsilon, \epsilon_{1}, T}\left(E_{\gamma}(0)\right) \int_{0}^{T}\left[|\phi|_{1, \Omega}^{2}+|\theta|_{1, \Omega}^{2}+\right. \\
\left.\left|\operatorname{curl} q_{t}\right|_{0, \Omega}^{2}\right] d t+C_{\gamma} E_{\gamma}(0)+C_{T} \epsilon_{1} E_{\gamma}^{2}(0)
\end{array}
$$

and selecting $\epsilon_{1}$ suitably small so $C_{T} E_{\gamma}(0) \epsilon_{1} \leq 1$ gives the desired estimate in Lemma 3.6 . 


\subsubsection{Second Estimate and completion of the proof of Lemma 6}

Our next step is to obtain the estimate for the potential energy.

Lemma 3.7 Let $u, w, \theta, \phi$ be a regular solution to (1), (2). Then, for any $\epsilon>0$, $T>0$ the following estimate takes place:

$$
\begin{gathered}
\int_{0}^{T} E_{p}(t) d t \leq C \int_{0}^{T} E_{k, \gamma}(t) d t+ \\
C\left(E_{\gamma}(0)\right) \int_{0}^{T}|\theta|_{1, \Omega}^{2}+|\phi|_{1, \Omega}^{2}+\left|\operatorname{curl} q_{t}\right|_{0, \Omega}^{2} d t+C E_{\gamma}(0)
\end{gathered}
$$

\section{Proof:}

We shall use the following multipliers -test functions applied to the variational form (12) :

$$
\begin{gathered}
\xi \equiv u ; \quad \psi \equiv 1 / 2 w \\
\left|\int_{0}^{T}\left(u_{t t}+b \operatorname{curl} q_{t}, u\right)_{\Omega} d t\right| \leq\left.\left(u_{t}, u\right)_{\Omega}\right|_{0} ^{T}+\int_{0}^{T}\left|u_{t}\right|_{0, \Omega}^{2} d t+ \\
\epsilon \int_{0}^{T}|u|_{0, \Omega}^{2}+C_{\epsilon} \int_{0}^{T}\left|\operatorname{curl} q_{t}\right|_{0, \Omega}^{2} d t \\
(\mathcal{C}[\boldsymbol{\epsilon}(u)+f(\nabla w)], \boldsymbol{\epsilon}(u))_{\Omega}+1 / 2(\mathcal{C}[\boldsymbol{\epsilon}(u)+f(\nabla w)], \nabla w \times \nabla w)_{\Omega}= \\
(\mathcal{C} N(u, w), N(u, w))_{\Omega} \\
(\phi \nabla w, \nabla w)_{0, \Omega} \leq C|\phi|_{0, \Omega}|w|_{2, \Omega}^{2}
\end{gathered}
$$

hence

$$
\begin{array}{r}
\int_{0}^{T}(\phi \nabla w, \nabla w)_{0, \Omega} d t \leq C \epsilon \int_{0}^{T} E_{p}(t) d t+C_{\epsilon}\left(E_{\gamma}(0)\right) \int_{0}^{T}|\phi|_{0, \Omega}^{2} d t \\
\left|1 / 2 \int_{0}^{T}\left(w_{t t}-\gamma \Delta w_{t t}, w\right)_{\Omega} d t\right| \leq C E_{\gamma}(0)+1 / 2 \int_{0}^{T}\left|w_{t}\right|_{\Omega}^{2}+\gamma\left|\nabla w_{t}\right|_{\Omega}^{2} d t \\
\left|\int_{0}^{T}\left[(\nabla \phi, u)_{\Omega}-1 / 2(\nabla \theta, \nabla w)\right] d t\right| \leq \epsilon \int_{0}^{T}|u|_{0, \Omega}^{2}+|w|_{1, \Omega}^{2} d t+ \\
\quad C_{\epsilon} \int_{0}^{T}\left[|\nabla \phi|_{0, \Omega}^{2}+|\nabla \theta|_{0, \Omega}^{2}\right] d t \\
\leq \epsilon \int_{0}^{T} E_{p}(t) d t+C_{\epsilon} \int_{0}^{T}\left[|\nabla \phi|_{0, \Omega}^{2}+|\nabla \theta|_{0, \Omega}^{2}\right] d t
\end{array}
$$


Combining the results in the inequalities (76)- (81) with the variational form (12) we obtain:

$$
\begin{aligned}
& \int_{0}^{T} E_{p}(t) d t \leq C_{\gamma} E_{\gamma}(0)+\epsilon \int_{0}^{T} E_{p}(t) d t+\int_{0}^{T} E_{k, \gamma}(t) d t \\
& \quad+C_{\gamma, \epsilon}\left(E_{\gamma}(0)\right) \int_{0}^{T}\left[|\nabla \phi|_{0, \Omega}^{2}+|\nabla \theta|_{0, \Omega}^{2}+b\left|\operatorname{curl} q_{t}\right|_{0, \Omega}^{2}\right] d t
\end{aligned}
$$

Taking $\epsilon$ suitably small (less than one) gives the desired inequality in Lemma 3.7.

To complete the proof of Lemma 3.4 it suffices to apply the results of Lemmas 3.6 and 3.7. Applying the estimate of Lemma 3.6 with $\epsilon$ suitably small in Lemma 3.7 gives

$$
\int_{0}^{T} E_{p}(t) d t \leq C_{\gamma} E_{\gamma}(0)+C_{\gamma, T}\left(E_{\gamma}(0)\right) \int_{0}^{T}\left[|\nabla \phi|_{0, \Omega}^{2}+|\nabla \theta|_{0, \Omega}^{2}+b\left|c u r l q_{t}\right|_{0, \Omega}^{2}\right] d t
$$

One more application of inequality in Lemma 3.6 yields

$$
\int_{0}^{T} E_{\gamma}(t) d t \leq C_{\gamma} E_{\gamma}(0)+C_{\gamma, T}\left(E_{\gamma}(0)\right) \int_{0}^{T}\left[|\nabla \phi|_{0, \Omega}^{2}+|\nabla \theta|_{0, \Omega}^{2}+b\left|\operatorname{curl} q_{t}\right|_{0, \Omega}^{2}\right] d t
$$

Recalling dissipativity inequality in lemma 4 and applying (84) gives

$$
T E_{\gamma}(T) \leq C_{\gamma, T}\left(E_{\gamma}(0)\right) \int_{0}^{T}\left[|\nabla \phi|_{0, \Omega}^{2}+|\nabla \theta|_{0, \Omega}^{2}+b\left|\operatorname{curl} q_{t}\right|_{0, \Omega}^{2}\right] d t+C_{\gamma} E_{\gamma}(T)
$$

and taking $T$ large enough so $T>2 C_{\gamma}$

$$
E_{\gamma}(T)+E_{\gamma}(0) \leq C_{\gamma, T}\left(E_{\gamma}(0)\right) \int_{0}^{T}\left[|\nabla \phi|_{0, \Omega}^{2}+|\nabla \theta|_{0, \Omega}^{2}+b\left|\operatorname{curl} q_{t}\right|_{0, \Omega}^{2}\right] d t
$$

the above inequality combined with inequality in (84) leads to the conclusion in Lemma 3.4.

\subsubsection{Completion of the proof of the main Theorem 1.3}

Stabilizability estimate in Lemma 3.4 together with dissipativity inequality (lemma 7) give

$$
E_{\gamma}(T) \leq C_{\gamma, T}\left(E_{\gamma}(0)\right)\left[E_{\gamma}(0)-E_{\gamma}(T)\right]
$$

Hence

$$
E_{\gamma}(T) \leq \frac{C_{\gamma, T}\left(E_{\gamma}(0)\right)}{1+C_{\gamma, T}\left(E_{\gamma}(0)+1\right)} E_{\gamma}(0) \leq \rho_{\gamma}\left(E_{\gamma}(0)\right) E_{\gamma}(0) ; \rho_{\gamma}<1
$$

Standard nonlinear semigroup result gives the exponential rates claimed in Theorem 1.3 and valid for regular solutions. The uniqueness result established in Theorem 1.1 allows (see e.g. [30]) to extend the decay rates to all weak solutions. 


\section{References}

[1] F.Ammar Khodja and A.Benabdallah. "Sufficient conditions for uniform stabilization of second order equations by dynamical controllers", C.R. Acad.Sci, 323: 615-620, 1996 .

[2] G. Avalos and I. Lasiecka. "Uniform decay rates in nonlinear thermoelastic systems without mechanical dissipation", Conference on Optimal Control, Theory and Algorithms, Kluwer AP, 1998.

[3] G. Avalos and I. Lasiecka. "Exponential Stability of a Thermoelastic System without mechanical Dissipation", Rend. ISTIT.Mat. Univ. Trieste, XXVIII:128,1997 .

[4] G. Avalos and I. Lasiecka. "Exponential Stability of a Thermoelastic System without mechanical Dissipation", IMA Preprint nr. 1357, 1357, 1995.

[5] G. Avalos and I. Lasiecka. "Exponential Stability of a Thermoelastic Plates with free boundary conditions and without mechanical Dissipation", SIAM J. Mathematical Analysis, 29:155-182, 1998.

[6] A. Benabdallah. "Modelling of von Karman system with thermal effects", Prépublications de l'équipe de mathématiques de Besançon n99/05, 1999.

[7] A. Benabdallah and I.Lasiecka. "Exponential decay rates for a full von Karman system of dynamic thermoelasticity", Journal of Differential Equations, 160:5193,2000 .

[8] A. Benabdallah and A. Soufyane. "Stabilization and stability for thermoelastic systems", Prépublications de l'équipe de mathématiques de Besançon, 97/43 and to appear in Journal of Dynamical and Control Systems.

[9] A. Benabdallah and D. Teniou. "Exponential Stability of a von Karman model with Thermal efffects", Electronic Journal of Differential Equations, 7:1-13, 1998.

[10] A. Bensoussan and G. Da Prato and M.C. Delfour and S.K. Mitter. Representation and Control of Infinite Dimensional Systems, Vol. II, Birkhauser, Boston-Basel-Berlin, 1993.

[11] E. Bisongin and V. Bisongin and G.P. Menzala and E. Zuazua. "On the exponential stability for von Karman equations in the presence of thermal effects", Preprint .

[12] M. Bohm. "Remarks on Complex Interpolation of some Nonlinear Operators", Math. Nachr, 153:191-206, 1991.

[13] M. Bohm. "Global welllposedness of the dynamic von Karman Equations for generalized Solutions", Manuscript, 1994.

[14] M. Bradley and I. Lasiecka. "Local exponential stabilization for a nonlinearl;y perturbed von karman plate", Nonlinear Analysis, 18:333-343, 1992.

[15] Ph. Ciarlet and P. Rabie. "Les Equations de von Karman", Springer Verlag, 1982.

[16] G. Duvaut and J.L.Lions. "Les Inéquations en Mécaniques et en Physiques", Dunod, 1972. 
[17] A. Favini and I. Lasiecka. "Wellposedness and regularity of second order abstract equations arising in hyperbolic-like problrms with nonlinear boundary conditions", Osaka Journal of Mathematics, 32, 1995

[18] Favini, A. and M. A. Horn and I. Lasiecka and D. Tataru. "Global excistence,uniqueness and regularity of solutions to a von Karman system with nonlinear boundary dissipation", Differential and Integral Equations, 9:267-294, 1996

[19] P. Grisvard. "Caractérisation de quelques espaces d'interpolation", Archive Rational Mechanics and Analysis, 25:40-63, 1967.

[20] M. A. Horn and I. Lasiecka. "Uniform decay of weak solutions to a von Karman plate with nonlinear dissipation", Differential and Integral Equations, 7:885908, 1994.

[21] M. A. Horn and I. Lasiecka. "Global stabilization of a dynamic von Karman plate with nonlinear boundary feedback", Applied Mathematics and Optimization, 31:57-84, 1995.

[22] H. Koch. "Slow decay in linear thermoelasticity", Preprint SFB, 97-47, 1997.

[23] H. Koch and A. Stachel. "Global existence of classical solutions to the dynamical von Karman equations", Math. Methods in the Applied Sciences, 16:581-586, 1993.

[24] J.U.Kim. "On the energy decay of a linear thermoelastic bar and plate", SIAM J. Math. Anal, 23:889-899,1992.

[25] I. Lasiecka. "Control and Stabilization of Interactive Structures", Systems and Control in the Twenty-First Centure, Birhkauser, 22:245-263, 1997.

[26] I. Lasiecka. "Uniform stabilizability of a full von Karman system with nonlinear boundary feedback", SIAM J. on Control, 36, 1998.

[27] I. Lasiecka "Uniform decay rates for full von Karman system of dynamic thermoelasticity with free boundary conditions and partial boundary dissipation", Communications on Partial Differential Equations, 24,9-10:1801-1849,1999.

[28] I. Lasiecka and D. Tataru. "Uniform boundary stabilization of semilinear wave equations with nonlinear boundary damping", Differential and Integral Equations, 6:507-533, 1993.

[29] I. Lasiecka." Existence and uniqueness of the solutions to second order abstract equations with nonlinear and nonmonotone boundary conditions", Nonlinear Analysis, 23:797-823, 1994.

[30] J. Lagnese. "Boundary Stabilization of Thin Plates", SIAM, 1989.

[31] J. Lagnese and G. Leugering. "Uniform stabilization of a nonlinear beam by nonlinear boundary feedback", Journal of Differential Equations, 91:355-388, 1991.

[32] J. Lagnese. "Uniform asymptotic energy estimates for solutions of the equations of dynamic plane elasticity with nonlinear dissipation at the boundary", Nonlinear Analysis,16:35-54, 1991.

[33] J. Lagnese. "Modeling and stabilization of nonlinear plates", International Ser. Num. Math.,100:247-264, 1991. 
[34] J. Lagnese and J.L.Lions. "Modeling, Analysis and Control of Thin Plates", Masson,1988.

[35] G.Lebeau and E. Zuazua. "Sur la décroissance non uniforme de l'énergie dans les systèmes de la thermoélasticité linéaire", C.R. Acad. Sci. Paris, 324:409415,1997 .

[36] J.L.Lions. "Quelques Méthodes de Résolution des Problèmes aux Limites Non linéaires", Dunod, Paris, 1969.

[37] Z.Liu and S.Zheng. "Exponential stability of the Kirchhoff plate with thermal or viscoelastic damping", Quaterly Applied Math., LV:551-564, 1997.

[38] K. Liu and M. Renardy. "A note on the equation of thermoelastic plate", Applied Math. Letters, 8:1-6, 1995.

[39] Z. Liu and Z. Liu. "Exponential stability and analyticity of abstract thermoelastic systems", Z.Agnew.Math.Physics, 48:885-904, 1997.

[40] J. Puel and M. Tucsnak. "Boundary stabilization for the von Karman equations", SIAM J. on Control, 33:255-273, 1996.

[41] J.E.M. Rivera. "Energy decay rate in linear thermoelasticity", Funkcial.Ekvac., 35:19-30, 1992.

[42] J.E.M. Rivera and R. Racke. "Smoothing properties,decay and global existence of solutions to nonlinear coupled sytems of thermoelastic plates", SIAM J. Math. Anal., pages1547-1563, 1995.

[43] V. I. Sedenko. "On uniqueness of the generalized solutions of initial boundary value problem for Marguerre-Vlasov nonlinear oscillations of the shallow shells", Russian Izvestiya, North-Caucasus Region, Ser. Natural Sciences, 1-2, 1994.

[44] I. Vorovic. "On some direct methods in nonlinear theory of vibration of curved shells", Izv. Akad. Nauk. SSSR. Mat., 21:747-784, 1957. 